\title{
A scanning Hartmann focus test for the EUVI telescopes aboard STEREO
}

\author{
R. Ohl*, S. Antonille*, D. Aronstein*, B. Dean*, M. Delmont*, J. d'Entremont**, W. \\ Eichhorn*, B. Frey*, S. Hynes^, D. Janssen**, D. Kubalak*, K. Redman@, R. Shiri*, J. \\ S. Smith*, P. Thompson\#, M. Wilson \\ ${ }^{a}$ NASA Goddard Space Flight Center, Greenbelt Maryland \\ cManTech International
}

\begin{abstract}
The Solar TErrestrial RElations Observatory (STEREO), the third mission in NASA's Solar Terrestrial Probes program, was launched in 2006 on a two year mission to study solar phenomena. STEREO consists of two nearly identical satellites, each carrying an Extreme Ultraviolet Imager (EUVI) telescope as part of the Sun Earth Connection Coronal and Heliospheric Investigation instrument suite. EUVI is a normal incidence, $98 \mathrm{~mm}$ diameter, Ritchey-Chretien telescope designed to obtain wide field of view images of the Sun at short wavelengths $(17.1-30.4 \mathrm{~nm})$ using a CCD detector. The telescope entrance aperture is divided into four quadrants by a mask near the secondary mirror spider veins. A mechanism that rotates another mask allows only one of these sub-apertures to accept light over an exposure. The EUVI contains no focus mechanism. Mechanical models predict a difference in telescope focus between ambient integration conditions and on-orbit operation. We describe an independent check of the ambient, ultraviolet, absolute focus setting of the EUVI telescopes after they were integrated with their respective spacecraft. A scanning Hartmann-like test design resulted from constraints implied by the EUVI aperture select mechanism. This inexpensive test was simultaneously coordinated with other NASA integration and test activities in a high-vibration, clean room environment. The total focus test error was required to be better than $+/-0.05 \mathrm{~mm}$. We describe the alignment and test procedure, sources of statistical and systematic error; and then the focus determination results using various algorithms. The results are consistent with other tests of focus alignment and indicate that the EUVI telescopes meet the ambient focus offset requirements. STEREO is functioning well on-orbit and the EUVI telescopes meet their on-orbit image quality requirements.
\end{abstract}

Keywords: alignment, focus, Hartmann, STEREO, SECCHI, EUVI, scanning Hartmann, ultraviolet

\section{INTRODUCTION}

NASA/Goddard Space Flight Center (GSFC) personnel from the Optics Branch (Code 551) and ManTech International Corp.'s GSFC Optics Function made an independent measurement of the STEREO/SECCHI/EUVI instruments' ambient "focus offset" during the Dec/Jan 2006 comprehensive performance test (CPT). The "EUVI Focus Test Results" memo defines this focus offset (Wuelser/LM, Jan 2006). From that document, the tolerance range for the EUVI focus is as follows:

$\begin{array}{ll}\text { - Requirement } & 0.22 \pm 0.35 \mathrm{~mm} \\ \text { - Goal } & 0.22 \pm 0.15 \mathrm{~mm}\end{array}$

The results of the GSFC EUVI Focus Test are listed in Table 1. These results are consistent with instruments that meet their focus offset requirements for ground testing.

Table 1. Summary of GSFC EUVI Focus Test results.

\begin{tabular}{|l|l|l|}
\hline Instrument & Focus offset (mm) & Test date \\
\hline
\end{tabular}




\begin{tabular}{|l|l|l|}
\hline EUVI-A & $0.49 \pm 0.01$ & 29 Dec 2005 \\
\hline EUVI-B & $0.43 \pm 0.01$ & 3 Jan 2006 \\
\hline
\end{tabular}

The quoted error contains contributions from both statistical and systematic sources in both the data collection and reduction/analysis. The error is discussed in Sections 2.0 and 3.0. One source of error not well covered in this work is the systematic errors associated with our centroiding technique used to produce the results shown in the above table. A more detailed analysis of this fitting technique could increase the size of the quoted error.

There are many different ways to determine best focus, so we examined about half a dozen (Section 3.8). The project should choose which is most relevant for comparison to science requirements and/or StimTel results. A great deal of effort went into the estimation of the uncertainty for these various methods (but perhaps less effort for the technique quoted in the summary table above). In particular, the error for the EUVI-A result should be larger than the EUVI-B result, because less data were obtained. This effect is evident in the data shown in Tables 3 and 4 below.

The uncertainties listed in the above summary table are not combinations of uncertainties from the results of different analysis methods. That is left to the reader, if that exercise is valuable. Note that results from the encircled energy technique more closely match StimTel results from the same time period (see the summary in Section 3.8).

These results are based on the "minimum area" focus criteria as described in Section 3.0 below. This metric is indicative of a "best" focus offset for the four EUVI entrance apertures considered together. However, other best focus criteria for the system give similar but significantly different results. The results of data reduction and analysis using other metrics are presented in Section 3.0. 


\subsection{Data collection}

We measured the ambient, ground-test focus offset for each of the EUVI instruments using a "scanning Hartmann test," similar to the test described in Ref. 1, using the EUVI entrance aperture selection mechanism as the scanning mechanism and a full-aperture GSE collimator as the source of the input wavefront.

The concept at the core of this measurement is that a simple, high-quality collimator provides a good measurement of the flight telescope's focus offset, given that the test is well-modeled and all systematic errors are understood. The placement of the pinhole light source at the collimator "zero focus" location (or, at least, a known focus location) was vital to this approach, where we define "focus" as the magnitude of a specific term in an orthonormal set of polynomials fit to a measured system wavefront error (WFE; e.g., Zernike expansion). We employed a commercial, Fizeau interferometer used in a "cat's eye" configuration with the collimator and a small, flat mirror at the system focus. The mirror contained a small pinhole in its coating, which we could back-illuminate using a fiber source. After the flat mirror and pinhole were placed at the focus of the GSE collimator using the interferometer's WFE signal, we illuminated EUVI with the collimator and pinhole source, stepping the pinhole through focus using a precision stage, and reading out image data from the flight telescope's detector at each defocus location for all four EUVI aperture quadrants. This gave us the micrometer stage offset on the collimator that made the images from the four off-axis telescope apertures appear smallest and/or "cross" through focus. The difference between this collimator offset and the collimator zero focus location gives the flight telescope focus offset via a straightforward multiplication of the ratio of the telescope-to-collimator focal lengths, squared $(0.1465$, in this case).

This section describes the equipment and technique (see references $2-4$ for more detail) and the statistical and systematic sources of error from the setup.

\subsection{GSE requirements}

We imposed the following requirements on our ground support equipment (GSE) for the GSFC EUVI Focus Test:

a. The collimator must permit a measurement of absolute EUVI focus offset to $\pm 0.05 \mathrm{~mm}$ or better. This value was chosen to match or improve upon StimTel measurements of EUVI absolute focus, which have an uncertainty of $\pm 0.05-$ $0.1 \mathrm{~mm}$ or so.

b. The collimator must be able to test EUVI instruments on both spacecraft.

c. The collimator must be able to test EUVI in the SCA environment during integration and test (I\&T) CPT with little interruption to the baseline I\&T flow (best effort). The optical axis of the test is $\sim 6.5 \mathrm{ft}$. above the SCA floor and is horizontal with respect to gravity.

d. The collimator optical design must be simple (i.e., readily model-able using a ray trace software) and its as-built wavefront error and best focus location must be measured. 
e. The collimator must be compatible with and use a short-wavelength (UV) source for small image size (best effort).

f. The collimator must be dimensionally stable in the SCA environment ( $\sim 5 \mathrm{deg} \mathrm{C}$ spatial and temporal temperature gradients).

g. The collimator must meet STEREO contamination control requirements for GSE.

\subsection{Work plan}

Details of the test plan and procedure can be found in references $2-4$.

The following work plan briefly summarizes the steps that we took to implement the GSFC EUVI Focus Test:

a. We built and characterized a GSE collimator assembly in Bldg. 7, room 013 using a large aperture commercial interferometer (18inch Zygo Corp. Mark IV/GPI ca. 1998 ) in a class $\sim 10,000$ environment. We also developed the GSE focus set procedure in this lab.

b. We moved the GSE to the SCA and mounted it at the approximate test height $(\sim 6.5 \mathrm{ft}$.$) and location.$

c. We re-characterized the GSE in the SCA using a commercial interferometer (6inch Zygo Corp. Verifire ca. 2003) in the sub-aperture of the collimator that EUVI will sample.

d. We checked the GSE focus set algorithm in the SCA environment at the test location.

e. We performed the focus test on EUVI:

i. We set the focus of the GSE collimator and characterize collimator wavefront error (WFE).

ii. We de-floated the air isolators on the optical table.

iii. We moved the interferometer away from the EUVI line-of-sight.

iv. We opened the EUVI aperture door and activated collimator's UV source.

v. We stepped the collimator pinhole in focus, obtaining EUVI images at each focus location for each EUVI sub-aperture.

vi. We replaced the interferometer, re-floated the table and re-measured the collimator focus.

f. We performed post-test characterization of the 6inch interferometer using NISTand Zygo-traceable reference flats.

g. We reduced the data.

h. We analyzed the data for multiple best focus metrics.

i. We produced a final report.

\subsection{GSE description}

Details of the GSE hardware can be found in references $2-4$.

The GSE is described in Figures 1-5.

\subsection{Test method notes and error discussion}

Details of the test plan and procedure can be found in references $2-4$. 
We align the collimator to the EUVI optomechanically, using theodolite metrology to establish Rx, Ry, X and Y location of the collimator with respect to the EUVI. This method aligned the collimator to within arcseconds of the center of the EUVI field of view (FOV) and produced knowledge of the location of the center of the EUVI aperture with respect to the center of the 6inch Zygo to about \pm 0.25 inch. Alignment of the collimator-Zygo-pinhole configuration was accomplished with the Zygo interferometer and an alignment telescope.

Details of some of the critical or novel steps in the above work plan are as follows: Step 2.2.a. We characterized the full aperture WFE of the GSE collimator in autocollimation using the 18inch Zygo and a small sub-aperture of a high-quality, concave, f/6 Zygo reference sphere mirror (MSER\# 5Q7). The result of this test is shown in Figure 6.

Step 2.2.e.i. We characterized the WFE of the GSE collimator in the EUVI-sampled subaperture using the 6inch Zygo and a high-quality flat mirror in retro-reflection (i.e., "cat's eye"). Only a part of one quadrant of the collimator is tested, since that is what EUVI samples during the UV focus test. Since this test is performed at cat's eye, some aberrations are not detected, because non-rotationally symmetric aberrations cancel in this optical test configuration. However, symmetric terms like focus (power) and spherical aberration are well measured. The WFE in the EUVI sub-aperture from this test is shown in Figure 7.

Step 2.2.e.i. In the above test configuration, we step the fat mirror at cat's eye through focus, measuring the change in the focus aberration (Zernike term) using a DC motor and stage with a repeatability of $\sim 0.3 \mu \mathrm{m}$ over a stroke of $\sim 50 \mathrm{~mm}$ (Physik Instrumente $\mathrm{GmbH})$. We define the "zero focus location for the collimator" using a linear, least squares fit to this data. In later, post-test analysis, we refine this zero focus location for the $98 \mathrm{~mm}$ EUVI sub-aperture of the 6inch diameter Zygo. This systematic effect results in the addition of $0.03 \pm 0.01 \mathrm{~mm}$ and $0.02 \pm 0.01 \mathrm{~mm}$ to the focus offset location for EUVIA and B, respectively (Section 3.0).

Step 2.2.e.v. The EUVI was operated with an exposure time of $70 \mathrm{~ms}$, FGS off and detector set to maximum gain. Note that the FGS actuator setting should not impact the focus measurement --- their state is mostly a field angle stability issue (i.e., with the FGS on, there is some question about image size stability). The detector's central $512 \times 512$ was read out at the highest pixel resolution. Dark exposures were obtained before and after the test with the aperture door closed. The brightness of the UV source $(355 \mathrm{~nm}$ laser) was adjusted such that the count rate was $<10,000$ counts in the peak pixel (i.e., the images were not saturated). Typically, four (4) images for each sub-aperture and focus location were obtained, for a total of 16 images per focus location. That is, four consecutive images were obtained for each sub-aperture position, before a new subaperture was tested.

The light source for the collimator is a $68 \mu \mathrm{m}$ diameter pinhole etched through the $\mathrm{Cr}$ coating on the surface of the small flat mirror located at cat's eye (i.e., the pinhole path is through fused silica glass). Since the pinhole is by definition in the surface of this highquality flat mirror, when the surface of the mirror is at the collimator's best focus location, the pinhole is in focus. The substrate of this flat mirror is transmissive in the 
UV. The figure error on this small flat is likely micron-level P-V (but was not measured). The fiber was single-mode in the UV.

The UV light source is a $355 \mathrm{~nm}$ laser (class IIIb). We operated this laser at a power of $\sim 10 \mathrm{~mW}$. We fed the laser to the pinhole using a $\sim 2 \mathrm{~m}$ length of $U V$-transmissive optical fiber.

Several independent steps were taken to ensure that the focus zero point of the collimator did not change during the table de-float and Zygo lift operations as well as during the test itself:

a. The cat's eye interferogram from the pinhole-mirror was monitored for changes during settling of the table (de-float). No significant changes were observed.

b. Dial indicators on the collimator primary mirror cell and pinhole assembly monitored the focus location of these components during the table de-float and later Zygo lift (accurate to $\sim 5 \mu \mathrm{m}$ ). No changes were observed.

c. Theodolites focus a finite conjugate on cross hair fiducials on the collimator primary mirror cell and pinhole assembly provided a non-contact method for monitoring the focus setting of these components, as well as a sanity check for the stability of the home motor position over the course of the test. No relative changes were observed, although small (e.g., $\sim 25 \mu \mathrm{m}$ ) rigid body motions of the collimator assembly were observed with these instruments with respect to the $\mathrm{SCA}$ room. During the test, when the focus motor reported to home position, its theodolite also verified that it was at home.

d. The temperature of the collimator assembly was monitored at 9 locations during the test setup and execution (Figure 8). The temperature data is shown in Figure 9 for both EUVI-A and -B tests. The collimator's secondary mirror magnification is large, but the secondary and primary mirrors are metered by Invar rods, which have a low CTE near room temperature $\left(-2 \times 10^{-6} /{ }^{\circ} \mathrm{C}\right.$, worst case; various published sources). Although the collimator's secondary-to-primary mirror separation was not independently measured during the test, the temperature data in Figure 9 indicate that the temperature changed by $<2.5^{\circ} \mathrm{C}$ during the EUVI-A test and $<1$ degC during the EUVI-B test. The data place a low limit on transient thermal gradients $(<<1 \mathrm{degC})$. This translates to a secondary-primary separation change of about 5 and $2 \mu \mathrm{m}$, respectively. The magnification of the secondary mirror is $\sim 4.5$ and the ratio of the telescope to collimator focal lengths gives 0.1465 . These factors yield about 15 and $6 \mu \mathrm{m}$ of uncertainty at the focus of EUVI-A and $-\mathrm{B}$, respectively. This is small compared to the net uncertainty on the final results quoted in the Executive Summary.

The focal plane of the wide-FOV EUVI is highly curved. Since the image from the collimator did not fall at the center of the EUVI detector, some focus offset due to field curvature is likely. The Lockheed-Martin (LM)-provided ZEMAX model shows a focal surface with a radius of curvature of $375.3 \mathrm{~mm}$ (concave toward the mirrors). In order to estimate this effect, we assume that the center of the detector is located at the optical axis of the as-built system as predicted by the ZEMAX model. If the image is located $\sim 111$ pixels or $\sim 1.5 \mathrm{~mm}$ from the center of the detector, this yields a focus offset of $\sim 3 \mu \mathrm{m}$ 
toward the EUVI mirrors. For the EUVI-A test, the spot location was $~ 100$ pixels from the center of the detector. For the EUVI-B test, the spot location was $<<100$ pixels from the center of the detector. Field curvature is therefore a negligible effect.

Sources of statistical error include photon "shot" noise and image motion due to vibration/acoustic loading and turbulence. The former was minimized by co-adding images of the bright source for maximum signal-to-noise ratio. The later effect caused image centroids to shift no more than $\sim 0.2$ pixel for temporally adjacent images.

\subsection{EUVI aperture registration}

It is critical that the X-Y location of the EUVI aperture within the collimator's tested aperture be known and the impact of any uncertainty quantified.

As mentioned above, the focus test uses an off-axis section of a Cassegrain telescope to feed a beam of varying power to EUVI by introducing light at a pinhole at a variety of focal positions. In order to calibrate the test, we use a Fizeau interferometer (6inch Zygo) to look into the target output aperture of the telescope. By using a reflective pinholemirror, we can orient the pinhole substrate such that the Zygo's output "retro-reflects" out the telescope and back into the Zygo. This retro-reflection or cat's eye return permits measurement of the WFE's defocus associated with the pinhole substrate with respect to the Zygo's reference flat surface. The raw optical path difference measured by the interferometer is twice the amount of defocus from light that originates at the pinhole because the light "double passes" the telescope in this calibration configuration (i.e., the optical path of the system is sampled twice).

During the test in the SCA, a linear fit to the measured defocus vs. motor position data was obtained using the full 6inch diameter aperture of the Zygo interferometer. This location was defined as the zero focus position or home for the collimator. However, this is not the same position as the zero focus position for the $98 \mathrm{~mm}$ sub-aperture sampled by EUVI. To determine this offset:

a. We determined the laboratory X-Y location of the EUVI boresight with respect to the collimator (after Rx, Ry alignment was achieved; Figures 10 and 11).

b. In post-processing, we determined the real zero defocus stage position for each EUVI sub-aperture.

c. We characterized the defocus knowledge as a function of stage position.

d. We combined defocus knowledge with static higher order wavefront aberrations within each sub-aperture.

e. We accounted for non-common path and other offsets.

f. We combined data from the above three steps to determine WFE input to EUVI as a function of stage position.

The results of this work are shown in Figures 12 and 13. Offsets are 0.03 and $0.02 \mathrm{~mm}$ for EUVI-A and $-B$, respectively, with a $1 \sigma$ error of $<0.01 \mathrm{~mm}$.

We could not perform both the interferometric test of the collimator and the EUVI test in situ, so the interferometer was removed from the setup for the EUVI image test. In order 
to check for any focus change during the test, we planned on replacing the interferometer after the EUVI image test and re-characterizing the focus location of the pinhole-mirror. For the test on EUVI-A, we ran out of time to complete this post-test check. For the test on EUVI-B, the focus motor failed near the end of the test, making a full sweep impossible. However, we had knowledge of the defocus location of the failed stage, so we replaced the Zygo and made a measurement of the gross power at that location $(\sim 19 \mathrm{~mm}$ from home). This measurement was consistent with expectations based on our analysis to $\sim 1 \sigma$. The exact nature of the focus stage failure is unknown, but is suspected to be due to wear from mechanical misalignments in the stage (not the motor) due to lack of lubrication (these stages and motors were originally built for vacuum far-UV optical applications --- FUSE heritage --- the stages are $>10$ years old). Our independent testing of the stage assembly prior to the EUVI Focus Test and monitoring of stage performance during the test with theodolites and dial indicators showed that there were no problems with the stage assembly before the failure. Furthermore, the encoders on the motors showed the absolute location of the stage in motor counts and all of our data reduction is in terms of motor counts (as opposed to commanded steps).

Note that we assume that the effective focal lengths of the GSE collimator and EUVI are per the design. We do not include any as-built data or uncertainties in the calculation of the ratio of those focal lengths. This could be a significant source of systematic error that would bias our results in the same direction.

Note that the non-orthogonality of the Zernike basis set used in this analysis over the obscured EUVI aperture is significant. However, the normalization of the individual Zernike terms is not important for this work.

The test setup was located $\sim 6.5 \mathrm{ft}$. above the floor of the SCA clean room and somewhat near the blower banks. This created great instability in the interferometric data, even with a vibrationally isolated optical table (vibration, acoustic loading, turbulence). This instability contributes greatly to the quoted error. In order to successfully gather interferometric data during the test, we abandoned the Zygo's standard phase-shifting algorithm for the company's proprietary FlashPhase ${ }^{T M}$ software, which supports the measurement of mid-to-low-quality optical systems with long optical path length in noisy environments. The data also underwent significant post-processing, including filtering to eliminate bad data from the focus offset calculation.

\subsection{Error summary}

The sources of systematic error and their impact are summarized in Table 2.

Table 2. Summary of systematic error.

\begin{tabular}{|l|c|c|l|}
\hline \multirow{2}{*}{ Error source } & \multicolumn{2}{|c|}{ Magnitude at EUVI image } & \multirow{2}{*}{ Note } \\
\cline { 2 - 3 } & EUVI-A & EUVI-B & \\
\hline Collimator focus set & $<0.01 \mathrm{~mm}$ & $<0.01 \mathrm{~mm}$ & $1 \sigma$ \\
\hline $\begin{array}{l}\text { SM-PM metering } \\
\text { (temp. change) }\end{array}$ & $<15 \mu \mathrm{m}$ & $<6 \mu \mathrm{m}$ & $\begin{array}{l}\text { Approximate upper } \\
\text { limit }\end{array}$ \\
\hline
\end{tabular}




\begin{tabular}{|l|l|l|l|}
\hline Field error & $\sim 3 \mu \mathrm{m}$ & $\ll 3 \mu \mathrm{m}$ & $\begin{array}{l}\text { Approximate upper } \\
\text { limit }\end{array}$ \\
\hline
\end{tabular}

\subsection{Data reduction, analysis and results}

We show the results seven (7) different best focus metrics and discuss them below. The results are summarized in Tables 3 and 4.

In general, images are dark-subtracted and artificially flat-fielded before analysis. The application of the latter depended on the sensitivity of the analysis to a flat field and only very low-order terms in the background were adjusted.

\subsection{Centroid area (method no. 1)}

The "centroid area" method is essentially the calculation of "where the spots cross" in focus. The centroid of each image is obtained via the fit of a two-dimensional, asymmetric Gaussian function (using a gradient-expansion algorithm to compute a nonlinear least squares fit; curvefit.pro, IDL, RSI, Inc.). For each focus position, the X-Y location of the peak of each sub-aperture's image results. For each focus position, the area between the X-Y centroid positions is approximated by calculating the length of the diagonals and multiplying these numbers.

Additional sources of error include the error in the Gaussian fit (small) and the error in the quadratic fit to the through-focus curve (dominant). In order to estimate the error (spread or repeatability) in this analysis, multiple co-added data sets were generated using the multiple sub-sets of the data obtained at each focus location.

The results are shown in Figures 14 and 15.

The StimTel results are most similar to this data reduction technique, but not exactly the same.

\subsection{Encircled energy of spot ensemble (method no. 2)}

The "encircled energy" method is the calculation of the encircled energy (i.e., synthetic circular aperture photometry) integrated over 4-18 pixel diameters on composite, 4aperture co-added images as a function of focus.

Additional sources of error include the choice of center for the circular aperture (via centroid; small effect) and the error in the quadratic fit to the through-focus curve (dominant). In order to estimate the error (spread or repeatability) in this analysis, multiple co-added data sets were generated using the multiple sub-sets of the data obtained at each focus location.

The results are shown in Figures 16 and 17. 


\subsection{Optical transfer function, OTF (method no. 3)}

The optical transfer function (OTF) is calculated through focus via the Fourier transform of the composite (ensemble), co-added 4-aperture image. The OTF is the spatial frequency response of the imaging system. These images are shown in Figures 18 and 19 for EUVI-A and $-\mathrm{B}$, respectively (linear grayscale plots).

Insufficient data near focus for the EUVI-A test prevented a quantitative measure of best focus using this technique, but results were obtained for EUVI-B.

Data pre-processing was used for this technique: (1) Four co-adds were used for each quadrant. (2) The background for each of these co-adds was then "fit" using linear " $x$ " and " $y$ " tilt terms using least squares optimization. (3) Noise filtering was then performed using a median filter and the results of steps (1)-(3) were co-added to produce a full-aperture data file at each focus position. The data was then registered to the center of the image array and cropped to a 64 by 64 pixel region about each co-added full aperture frame. The Fourier transform was then taken and the resulting power-spectra derived as the complex-conjugate square of the Fourier transform. These images are shown in Figures 18 and 19 for EUVI-A and -B, respectively (linear grayscale plots).

\subsection{Peak pixel (method no. 4)}

This image quality metric examines the brightness of the peak pixel in composite, coadded images that are normalized for total energy as a function of focus.

Insufficient data near focus for the EUVI-A test prevented a quantitative measure of best focus using this technique, but results were obtained for EUVI-B.

This image quality metric examines the brightness of the peak pixel in composite, coadded images consisting of data from each of the EUVI entrance pupil quadrants. Data pre-processing was used for this technique: (1) Four co-adds were used for each quadrant. (2) The background for each of these co-adds was then "fit" using linear " $x$ " and " $y$ " tilt terms using least squares optimization. (3) Noise filtering was then performed using a median filter and the results of steps (1)-(3) were co-added to produce a full-aperture data file at each focus position. The data was then registered to the center of the image array and the peak pixel values of each data-frame were recorded as a function of the measured defocus position. Finally, the peak-pixel values were least squares fit as quadratic function of the measured defocus position. Error bars for the method were derived using a standard variance analysis of the data values and least squares fit.

\subsection{Image sharpness (method no. 5)}

This image quality metric examines the spatial irradiance gradient across the spot in composite, co-added images that are normalized.

This image quality metric examines the spatial intensity gradient: 


$$
S=\sum_{j} \sum_{k}\left(\text { image }_{j k}\right)^{2} /\left(\sum_{j} \sum_{k} \text { image }_{j k}\right)^{2}
$$

across the spot in composite, co-added images that are normalized. Data pre-processing was used for this technique: (1) Four co-adds were used for each quadrant. (2) The background for each of these co-adds was then "fit" using linear " $x$ " and " $y$ " tilt terms using least squares optimization. (3) The data values were then normalized to the peak values of each quadrant, (4) Noise filtering was then performed using a median filter and the results of steps (1)-(4) were co-added to produce a full-aperture data file at each focus position. The data was then registered to the center of the image array and the $S$ values of each data-frame were recorded as a function of the measured defocus position. Finally, the $S$ values were least squares fit as quadratic function of the measured defocus position. Error bars for the method were derived using a standard variance analysis of the data values and least squares fit.

\subsection{Grid search, whole pupil (method no. 6)}

This is essentially a rough "phase retrieval" technique that uses simple assumptions about the uniformity of the intensity in the pupil plane to provide a quantitative estimate of defocus in the WFE as a function of collimator focus. This technique uses information from all 4 EUVI sub-apertures together in its algorithm.

This method uses a grid-search $\chi^{2}$ minimization "phase retrieval" technique. The approach is based on a forward Fourier transform model of the image data based on the assumption of a full-aperture entrance pupil. This technique uses information from all 4 EUVI sub-apertures together. To derive this error metric the $\chi^{2}$ is calculated as a function of the data and model as a function of defocus:

$$
\chi^{2}=\sum_{i} \sum_{j} \mid \text { data }_{i j}-\left.\operatorname{model}_{i j}(\text { defocus })\right|^{2}
$$

The $\chi^{2}$ function is then minimized to find each defocus value in waves at the each data point. Data pre-processing was used for this technique: (1) Four co-adds were used for each quadrant. (2) The background for each of these co-adds was then "fit" using linear " $x$ " and " $y$ " tilt terms using least squares optimization. (3) The data values were then normalized to the peak values of each quadrant. (4) Noise filtering was then performed using a median filter and the results of steps (1)-(4) were co-added to produce a fullaperture data file at each focus position. The data was then registered to the center of the image array and the $\chi^{2}$ function is then minimized to find each defocus value in waves at each measured defocus position (mm). Finally, the fitted defocus values in waves were least squares fit as quadratic function of the measured defocus position (mm). Error bars for the method were derived using a standard variance analysis of the data values and least squares fit. 


\subsection{Grid search, individual quadrants (method no. 7)}

Again, this is essentially a rough "phase retrieval" technique that uses simple assumptions about the uniformity of the intensity in the pupil plane to provide a quantitative estimate of defocus in the WFE as a function of collimator focus. However, this technique uses information from each EUVI sub-aperture individually.

This method also uses a grid-search $\chi^{2}$ minimization "phase retrieval" technique. The approach is based on a forward Fourier model of the image data. However, this technique uses information from each EUVI sub-aperture individually. To derive this error metric the $\chi^{2}$ is calculated as a function of the data and model as a function of defocus:

$$
\chi^{2}=\sum_{i} \sum_{j} \mid \operatorname{data}_{i j}-\operatorname{model}_{i j}\left(\text { defocus }\left.\right|^{2}\right.
$$

The $\chi^{2}$ function is then minimized to find each defocus value in waves at the each data point. Data pre-processing was used for this technique: (1) Four co-adds were used for each quadrant. (2) The background for each of these co-adds was then "fit" using linear " $x$ " and " $y$ " tilt terms using least squares optimization. (3) The data values were then normalized to the peak values of each quadrant. (4) Noise filtering was then performed using a median filter and the results of steps (1)-(4) were co-added to produce a fullaperture data file at each focus position. The data was then registered to the center of the image array and the $\chi^{2}$ function is then minimized to find each defocus value in waves at each measured defocus position $(\mathrm{mm})$. Finally, the fitted defocus values in waves were least squares fit as quadratic function of the measured defocus position (mm). Error bars for the method were derived using a standard variance analysis of the data values and least squares fit.

\subsection{Summary of results}

The results of these techniques are summarized in Tables 3 and 4 for EUVI-A and -B, respectively.

The centroid area method is most similar to a Hartmann-type test, essentially tracking the centroid of each spot through focus. The encircled energy criteria agrees well with the area technique for EUVI-A, but less well for EUVI-B. Where more data is available, the grid search techniques are also powerful indicators of defocus, yielding results that agree with the area technique to about $1 \sigma$. The image and OTF linear grayscale images showing in Figures 18 and 19 give a good qualitative measure of focus, also confirming the centroid area result. The results of these additional analysis approaches confirm the centroid area data reported in Section 1.0.

Note that much more effort went into the uncertainty analysis for methods $3-7$ vice methods $1-2$. Hence, the error bars are more realistic and appear more consistent with each other. 
Table 3. Summary of analysis results for EUVI-A.

\begin{tabular}{|l|l|l|}
\hline $\begin{array}{l}\text { Method } \\
\text { number }\end{array}$ & Method name & EUVI focus offset $(\mathbf{m m})$ \\
\hline 1 & Centroid area & $0.49 \pm 0.01$ \\
\hline 2 & Encircled energy & $0.43 \pm 0.06$ \\
\hline 3 & OTF & Insufficient data \\
\hline 4 & Peak pixel & Insufficient data \\
\hline 5 & Sharpness & $0.36 \pm 0.51$ \\
\hline 6 & Grid search, whole pupil & $0.30 \pm 0.27$ \\
\hline 7 & Grid search, quadrants & $0.30 \pm 0.12$ \\
\hline & $\begin{array}{l}\text { Weighted average of } \\
\text { methods 5-7 }\end{array}$ & $0.30 \pm 0.16$ \\
\hline
\end{tabular}

Table 4. Summary of analysis results for EUVI-B.

\begin{tabular}{|l|l|l|}
\hline $\begin{array}{l}\text { Method } \\
\text { number }\end{array}$ & Method name & EUVI focus offset (mm) \\
\hline 1 & Centroid area & $0.43 \pm 0.01$ \\
\hline 2 & Encircled energy & $0.33 \pm 0.03$ \\
\hline 3 & OTF & $0.39 \pm 0.18$ \\
\hline 4 & Peak pixel & $0.37 \pm 0.35$ \\
\hline 5 & Sharpness & $0.42 \pm 0.21$ \\
\hline 6 & Grid search, whole pupil & $0.45 \pm 0.05$ \\
\hline 7 & Grid search, quadrants & $0.42 \pm 0.04$ \\
\hline & $\begin{array}{l}\text { Weighted average of } \\
\text { methods 3-7 }\end{array}$ & $0.42 \pm 0.05$ \\
\hline
\end{tabular}




\subsection{References}

1. Introduction to Optical Testing (Geary, SPIE Optical Engineering Press, Volume TT 15, p. 88, 1993).

2. GSFC EUVI Focus Test Plan (Ohl, Dec 2005).

3. GSFC EUVI Focus Test Procedure (Ohl, Dec 2005).

4. GSFC EUVI Focus Test Laser Safety Plan (Redman, Dec 2005). 
CONFERENCE: SPIE 2007, OP07-OP112-12 (conference on opt sys eng and alignment, Sasian, Ruda)

TITLE: A scanning Hartmann focus test for the EUVI telescopes aboard STEREO

\author{
AUTHORS: R. Ohl*, S. Antonille*, D. Aronstein*, B. Dean*, M. Delmont*, J. \\ d'Entremont**, W. Eichhorn*, B. Frey*, S. Hynes^, D. Janssen**, D. Kubalak*, K. \\ Redman@, R. Shiri*, J. S. Smith*, P. Thompson\#, M. Wilson*
}

\title{
AFFILIATIONS:
}

*NASA/Goddard Space Flight Center

Greenbelt, MD 20771

${ }^{\wedge}$ Swales Aerospace, Inc.

Beltsville, MD 20705

@ManTech International

NASA/Goddard Space Flight Center

Greenbelt, MD 20771

\#Johns Hopkins University

Applied Physics Laboratory

Laurel, $\mathrm{MD}$

**Lenox Laser

12530 Manor Rd.

Glen Arm, MD 21057

Tel. 4105923106

\section{ABSTRACT:}

The Solar TErrestrial RElations Observatory (STEREO), the third mission in NASA's Solar Terrestrial Probes program, was launched in 2006 on a two year mission to study solar phenomena. STEREO consists of two nearly identical satellites, each carrying an Extreme Ultraviolet Imager (EUVI) telescope as part of the Sun Earth Connection Coronal and Heliospheric Investigation instrument suite. EUVI is a normal incidence, $98 \mathrm{~mm}$ diameter, Ritchey-Chretien telescope designed to obtain wide field of view images of the Sun at short wavelengths $(17.1-30.4 \mathrm{~nm})$ using a CCD detector. The telescope entrance aperture is divided into four quadrants by a mask near the secondary mirror spider veins. A mechanism that rotates another mask allows only one of these subapertures to accept light over an exposure. The EUVI contains no focus mechanism. Mechanical models predict a difference in telescope focus between ambient integration conditions and on-orbit operation. We describe an independent check of the ambient, ultraviolet, absolute focus setting of the EUVI telescopes after they were integrated with their respective spacecraft. A scanning Hartmann-like test design resulted from 
constraints implied by the EUVI aperture select mechanism. This inexpensive test was simultaneously coordinated with other NASA integration and test activities in a highvibration, clean room environment. The total focus test error was required to be better than $+/-0.05 \mathrm{~mm}$. We describe the alignment and test procedure, sources of statistical and systematic error, and then the focus determination results using various algorithms. The results are consistent with other tests of focus alignment and indicate that the EUVI telescopes meet the ambient focus offset requirements. STEREO is functioning well onorbit and the EUVI telescopes meet their on-orbit image quality requirements.

KEYWORDS: alignment, focus, Hartmann, STEREO, SECCHI, EUVI, scanning Hartmann, ultraviolet 


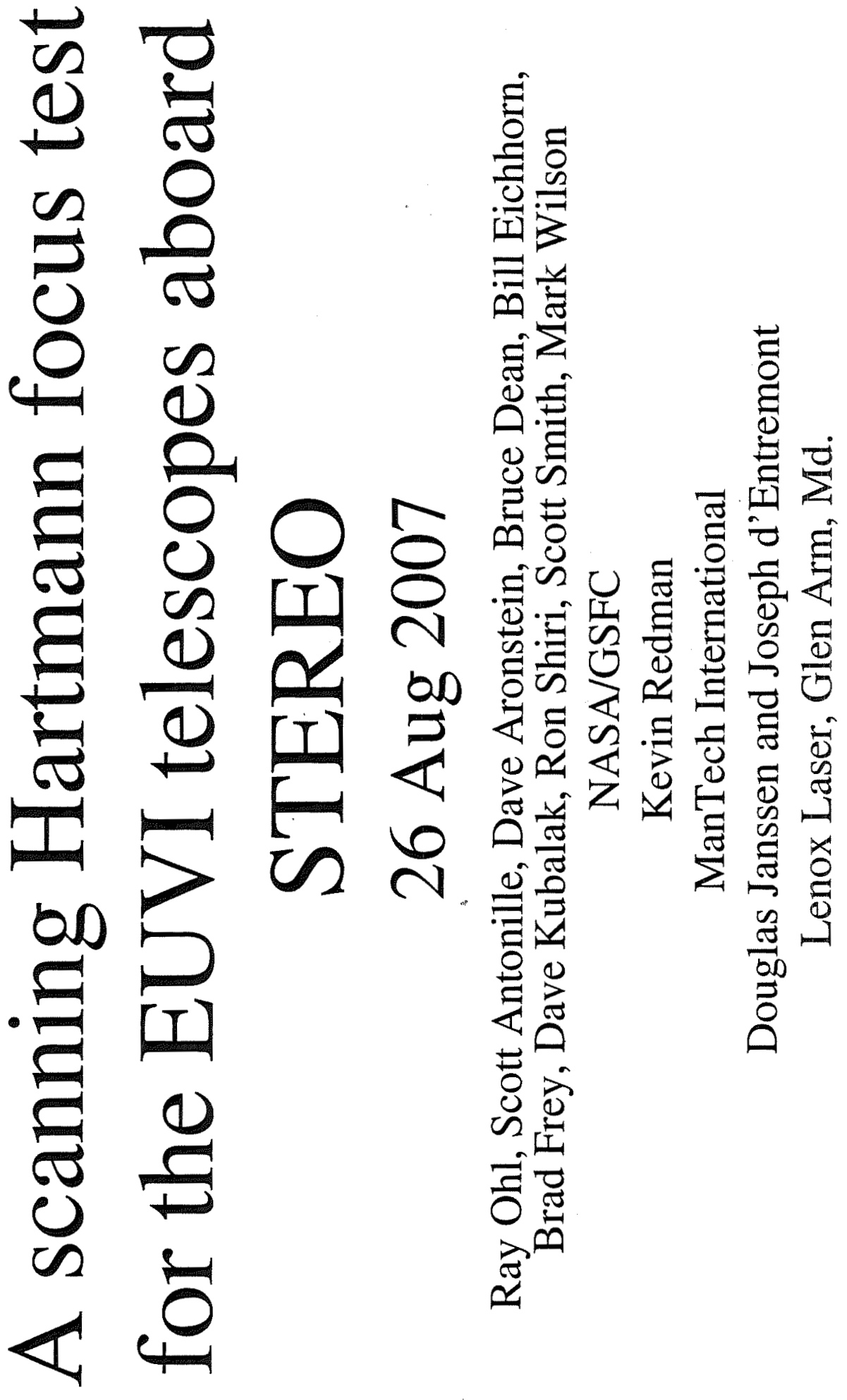




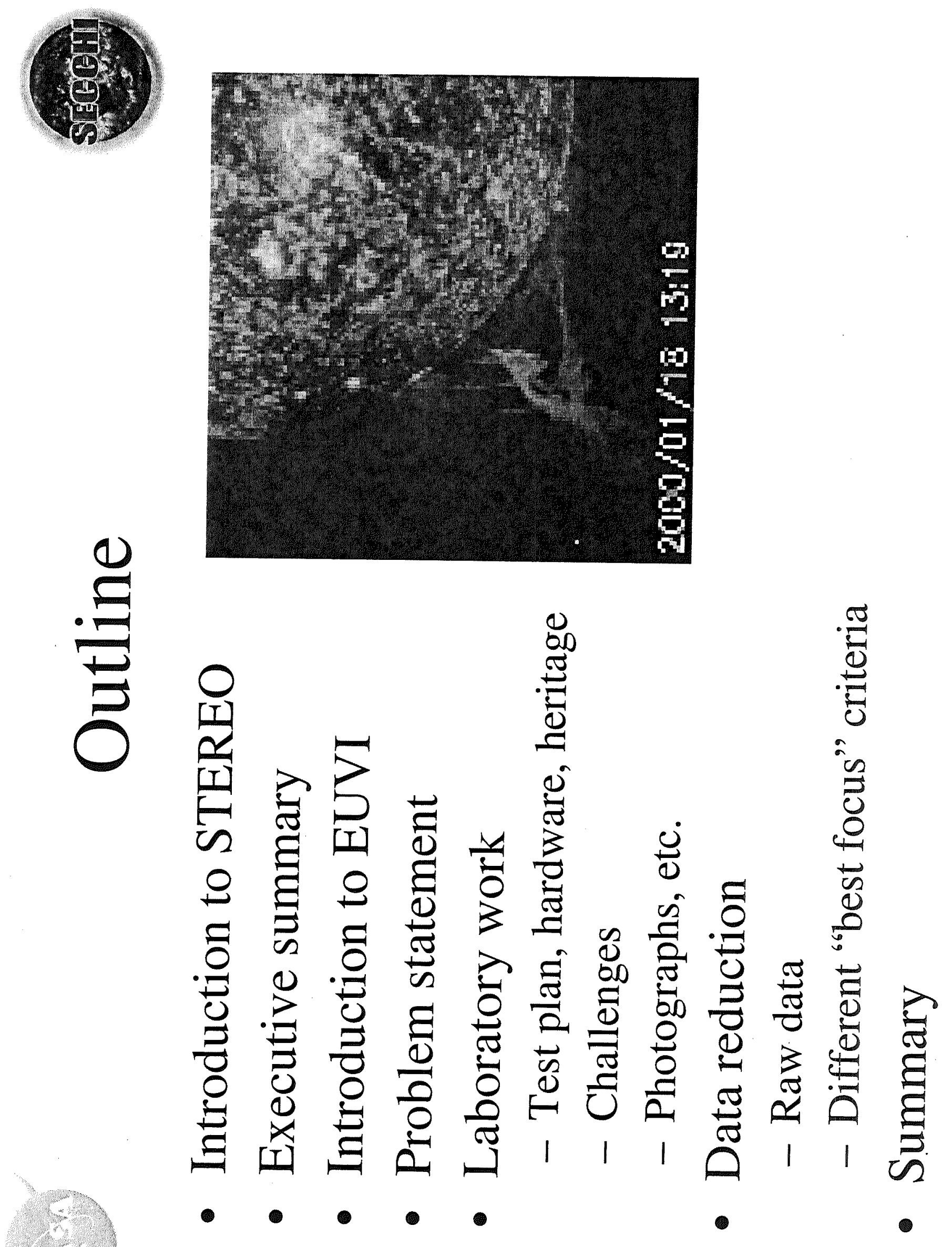

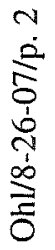




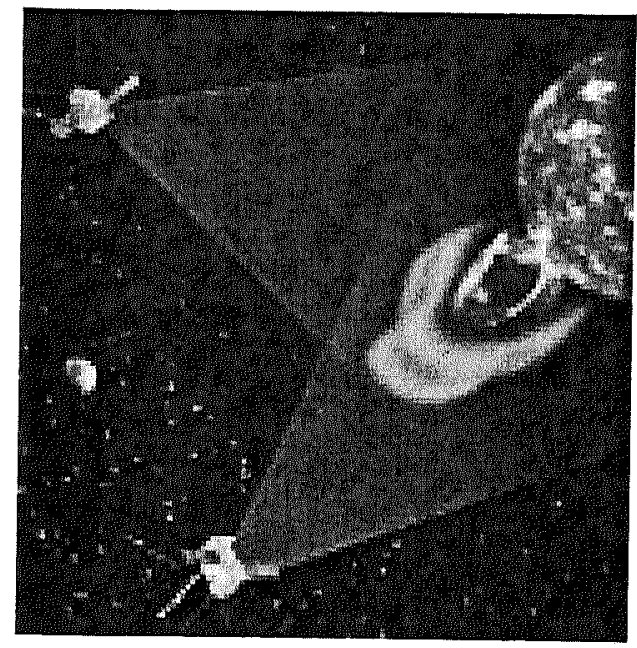

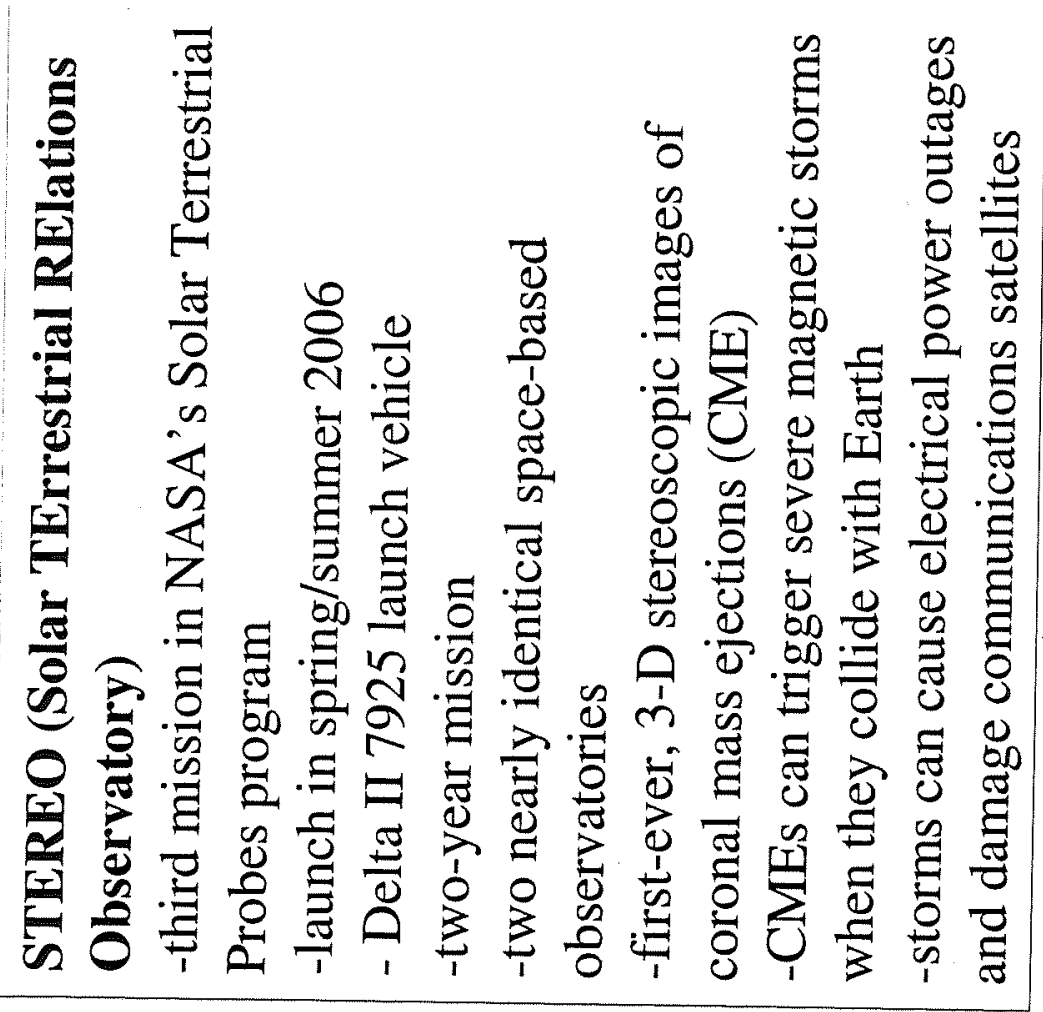

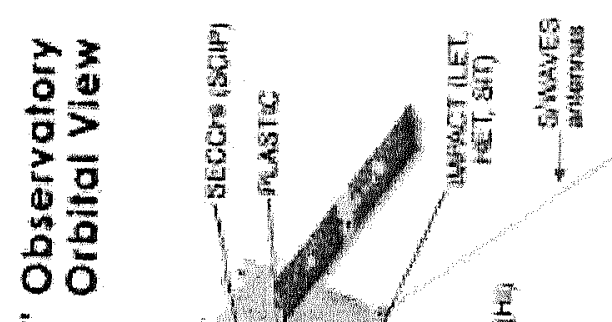
章
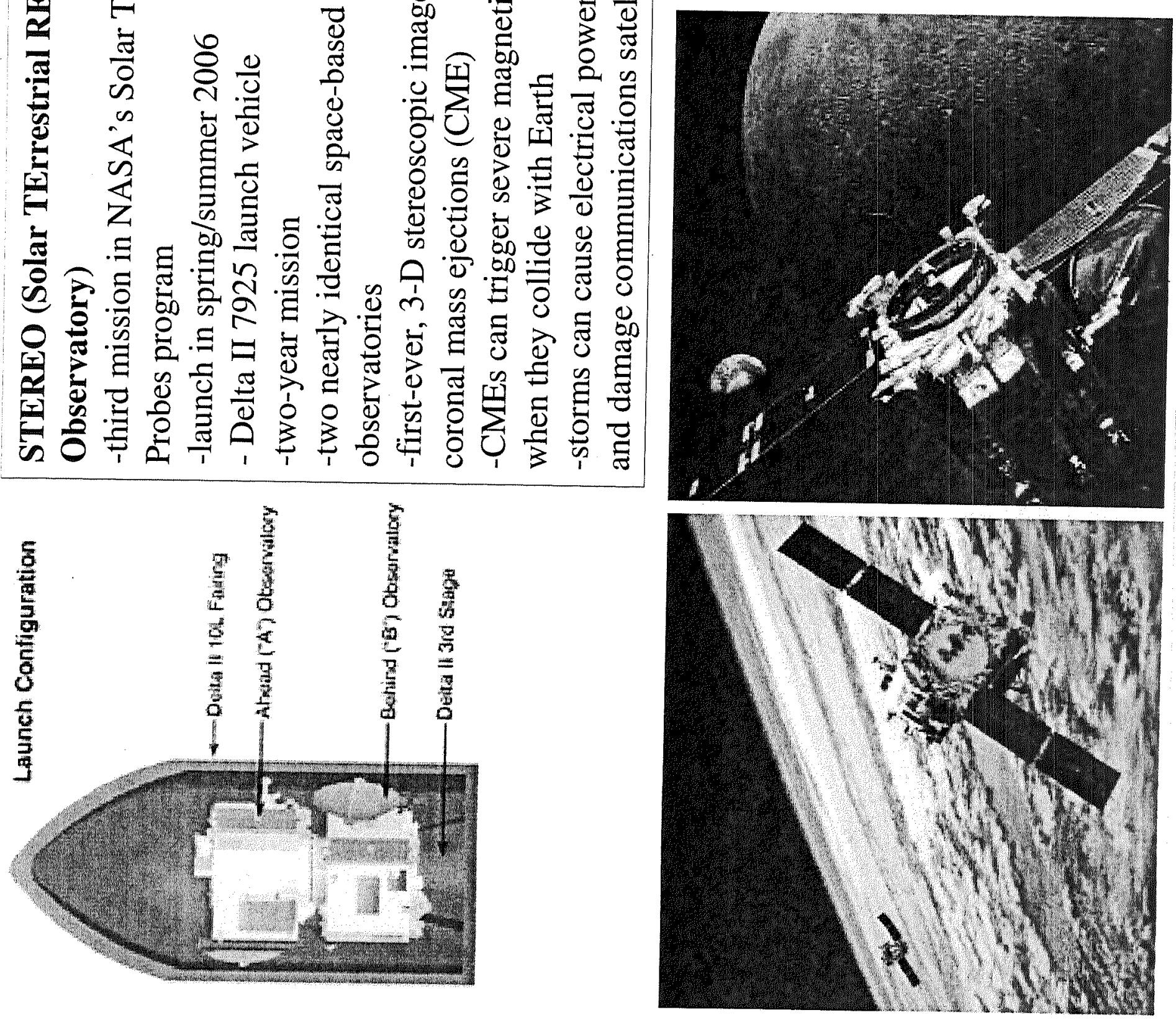


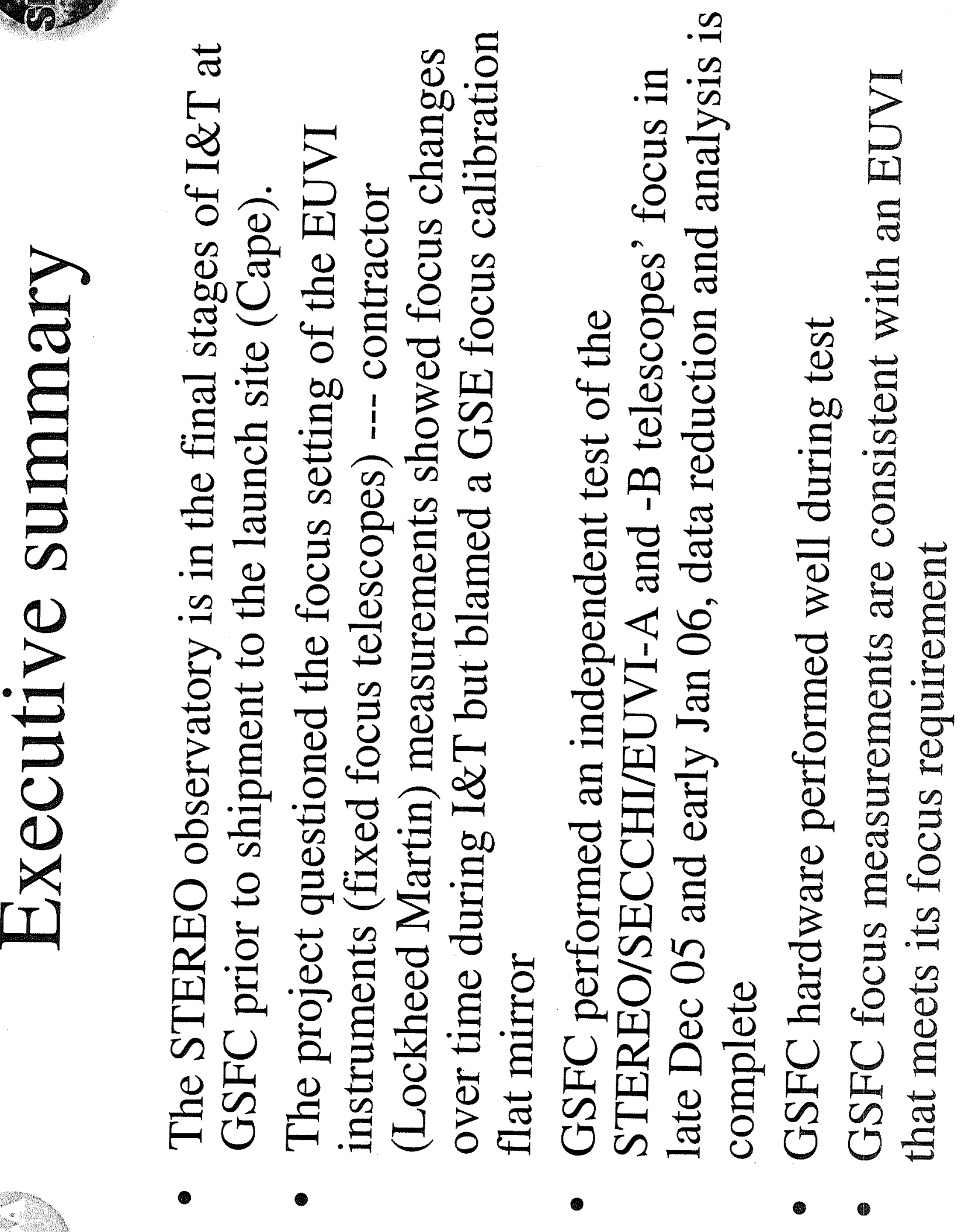

$\bar{\sigma}$

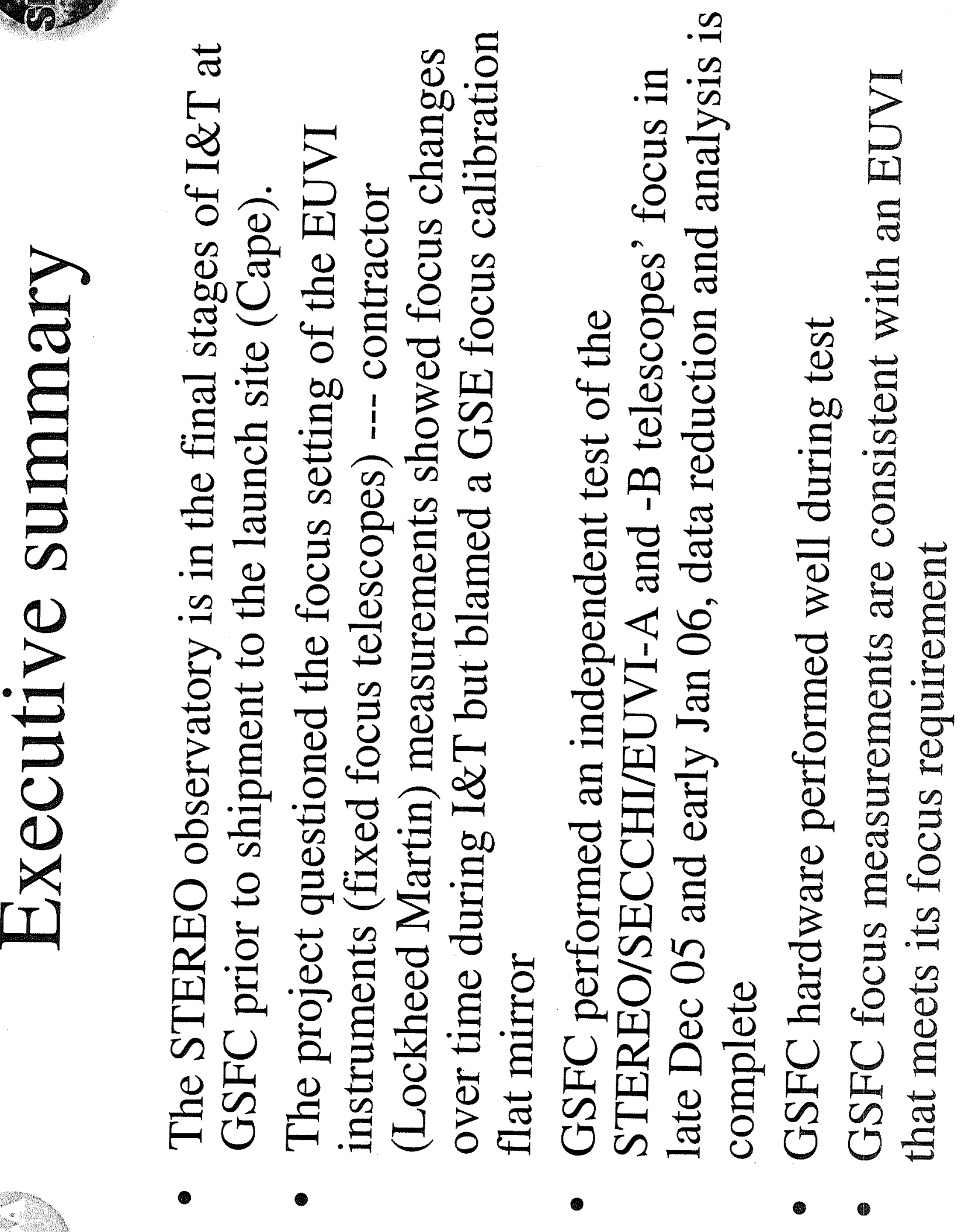




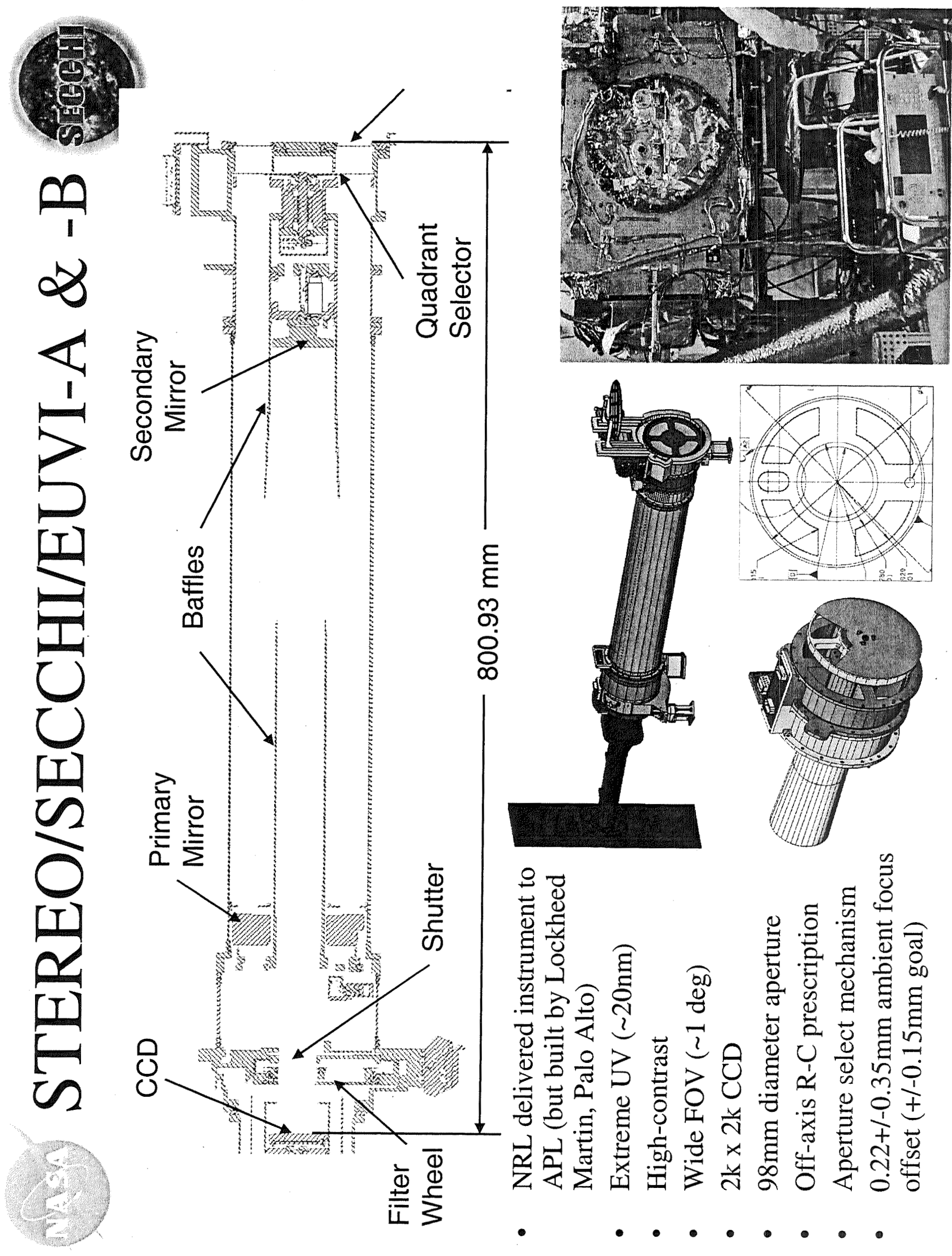



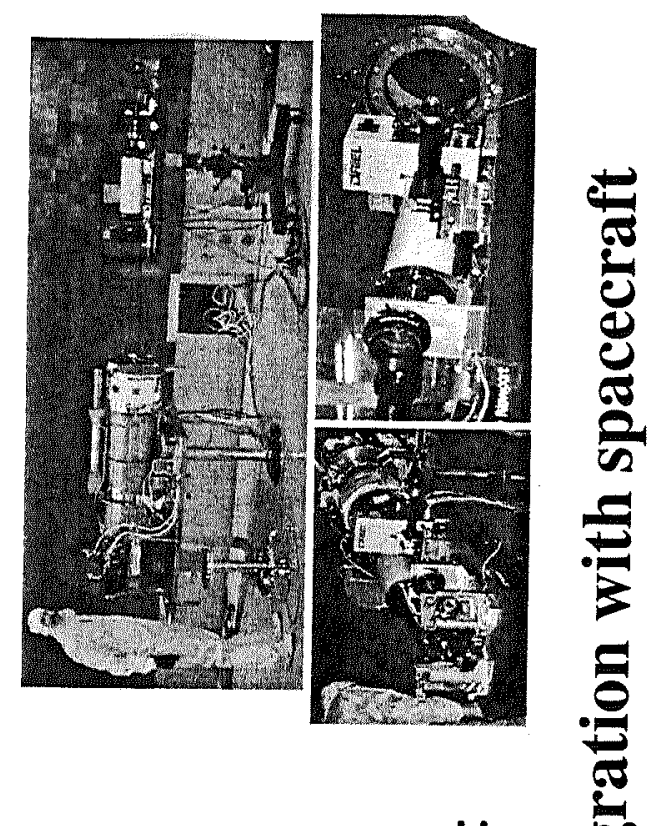

is

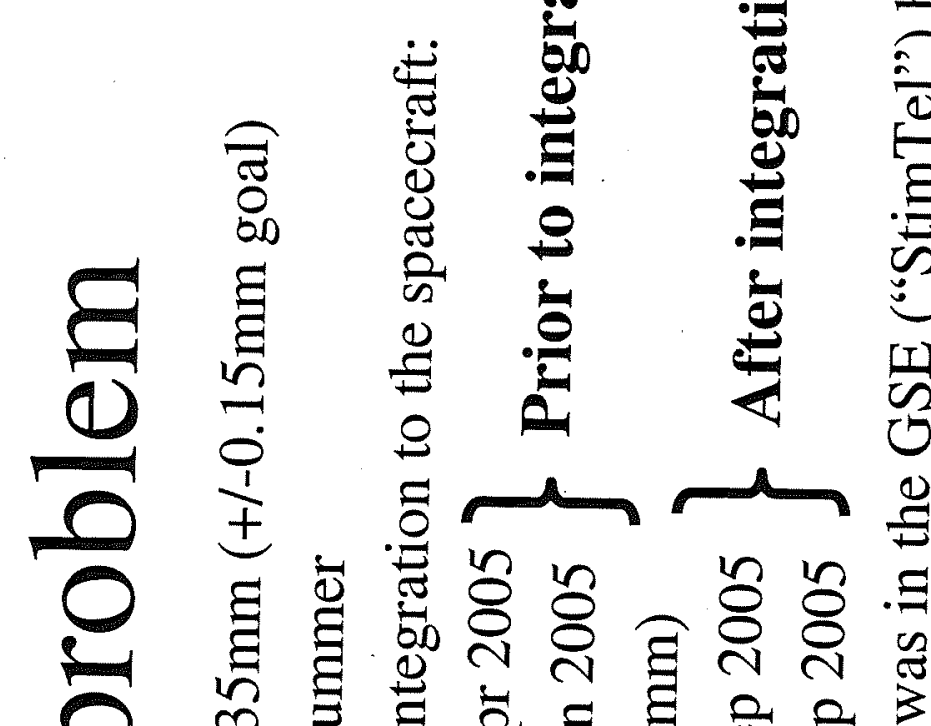

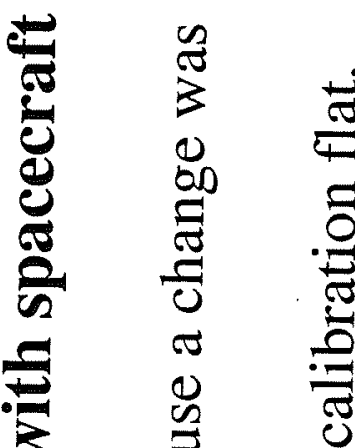

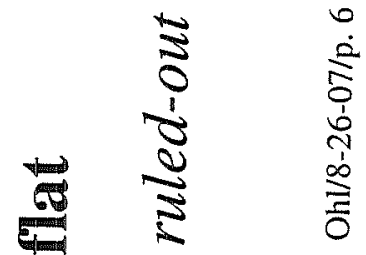

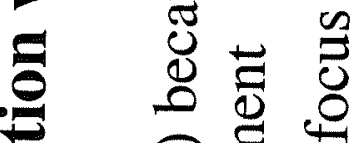

$\approx$ 응 की

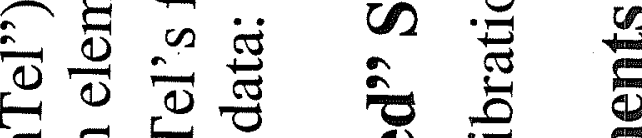

छ

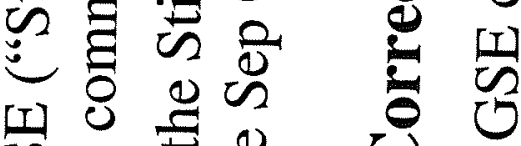

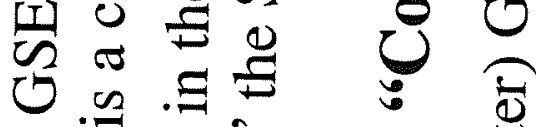

(1) 20

020

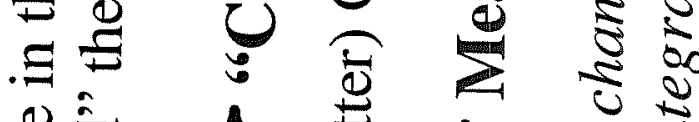
I II

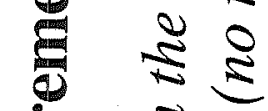

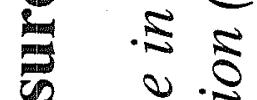

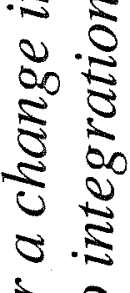

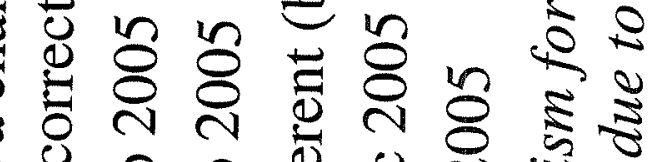

․

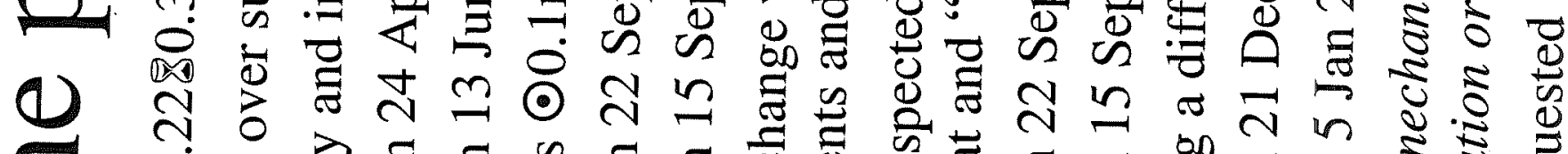

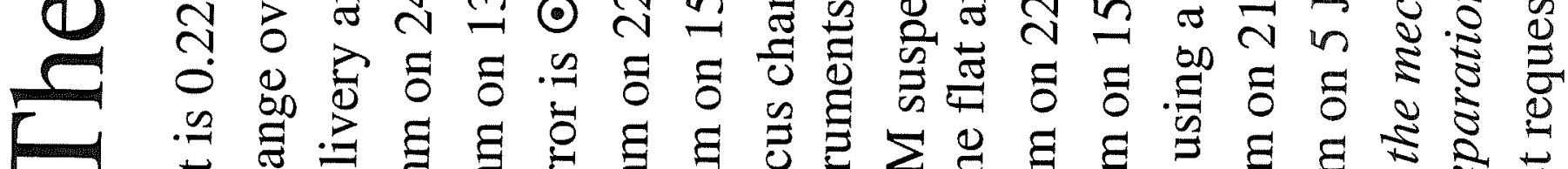

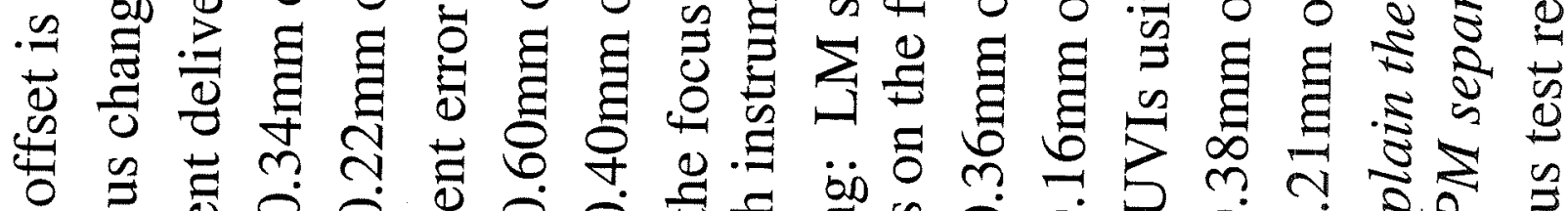
色

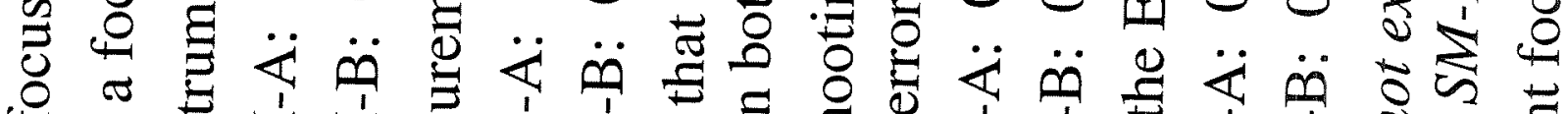

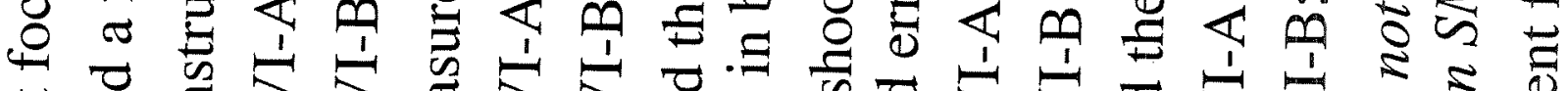
范导

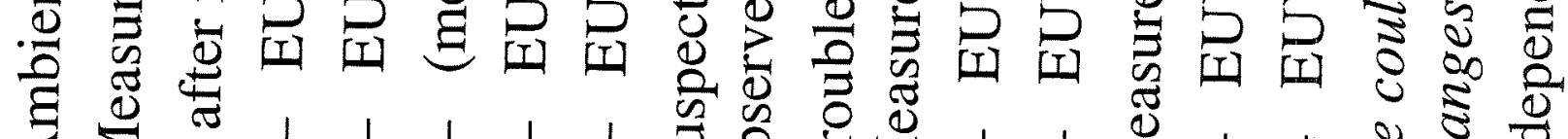

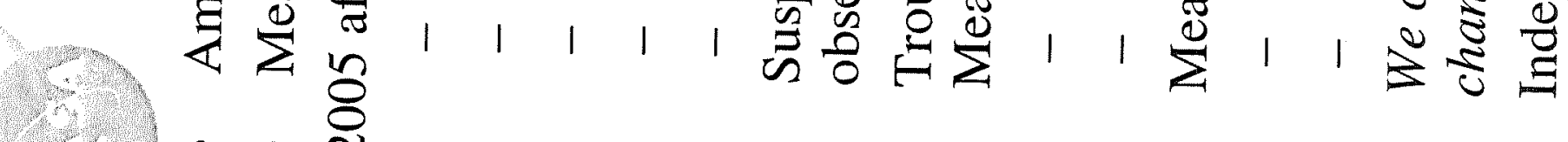
- $\bigodot_{0}$ 
$\overline{0}$

策

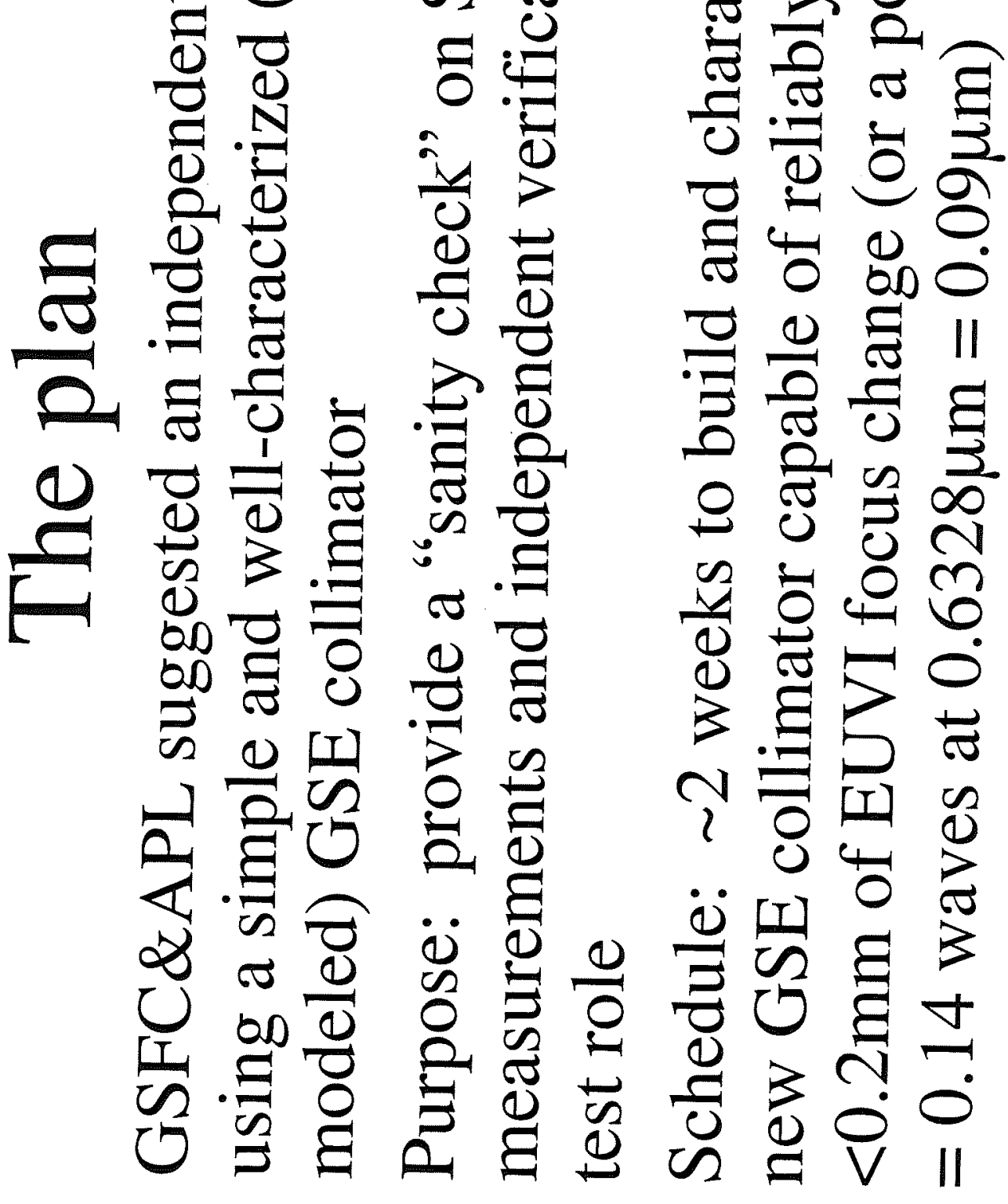

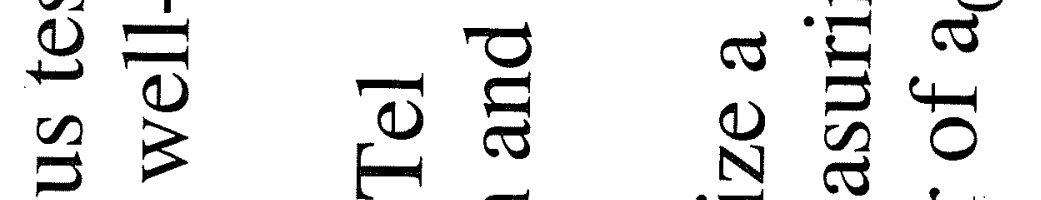

宅

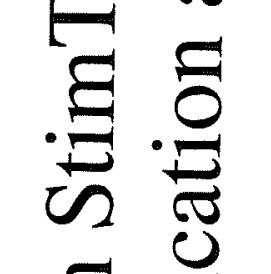

(1) $E$ B

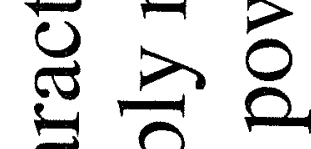

-

- 


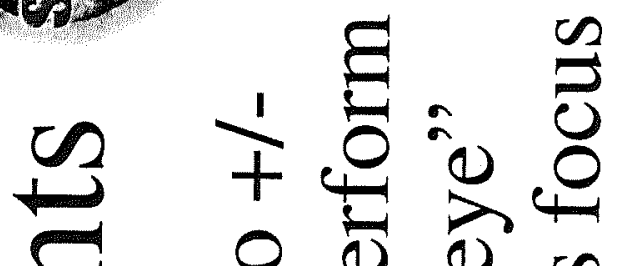

잉 0

(1) 3 an

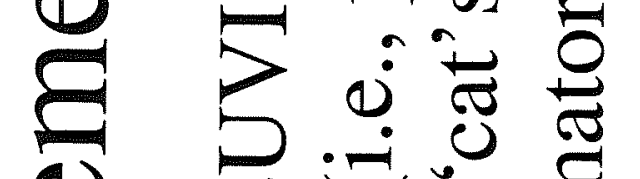

(1) II

.当岁离离号

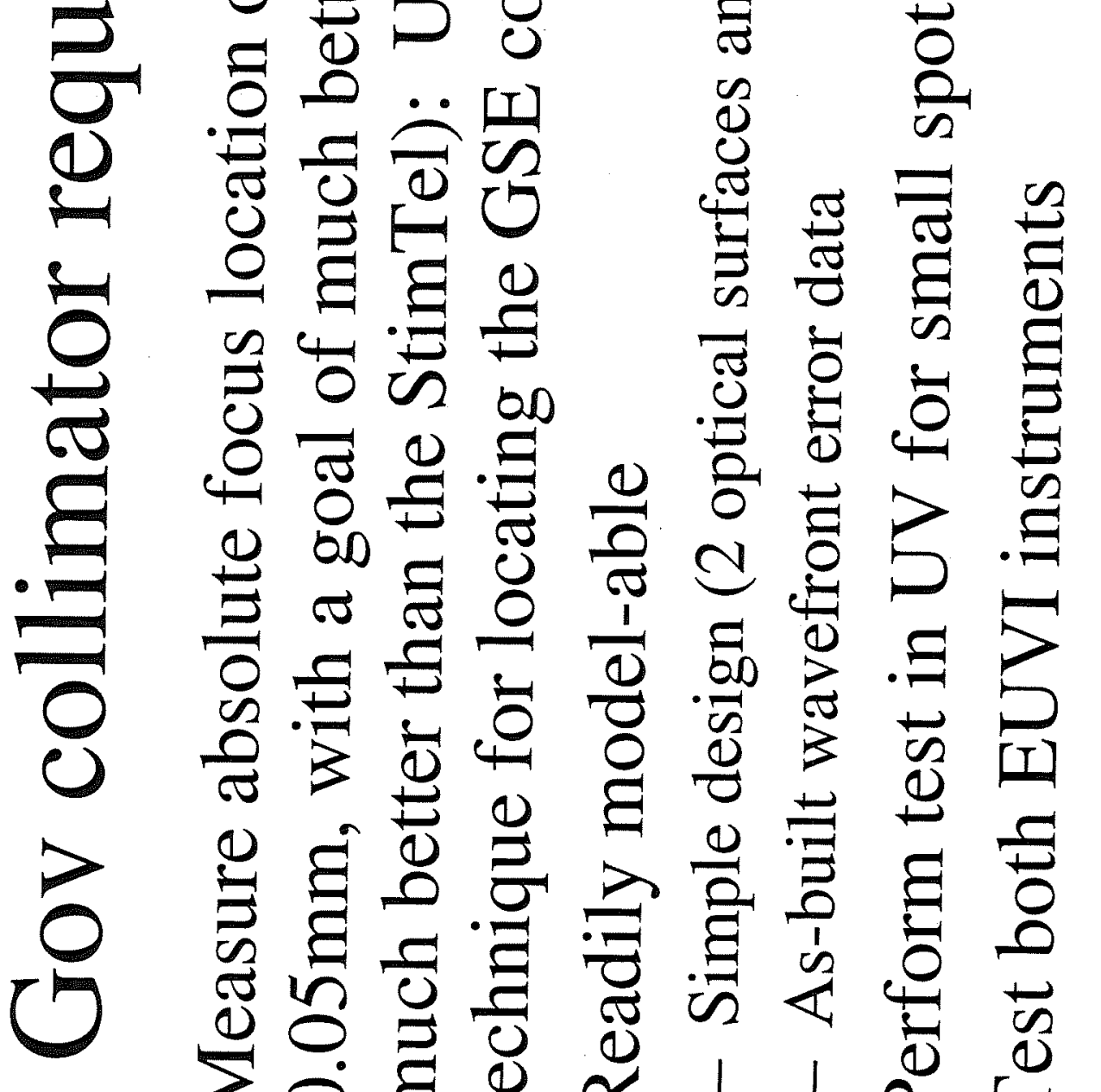

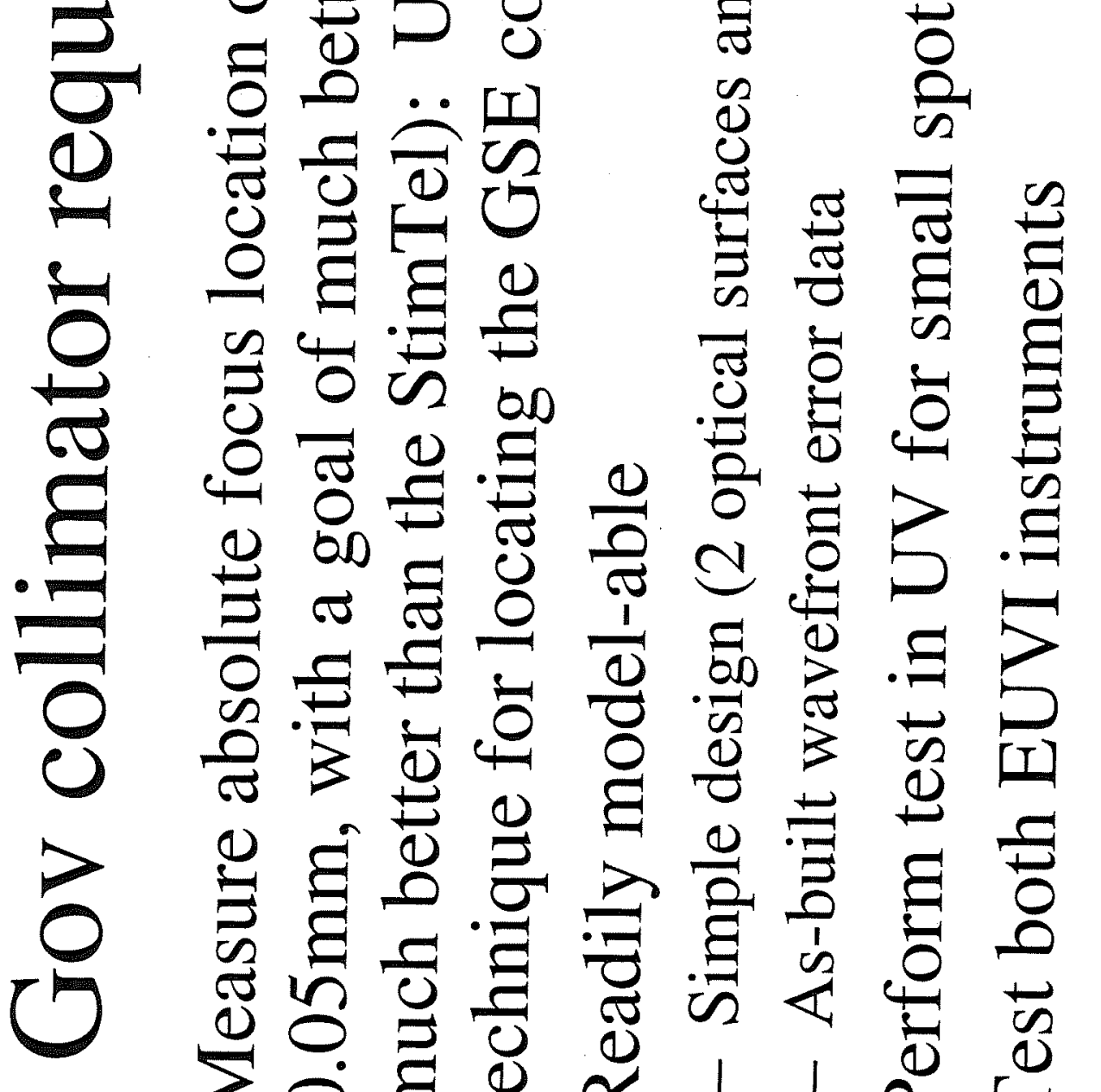

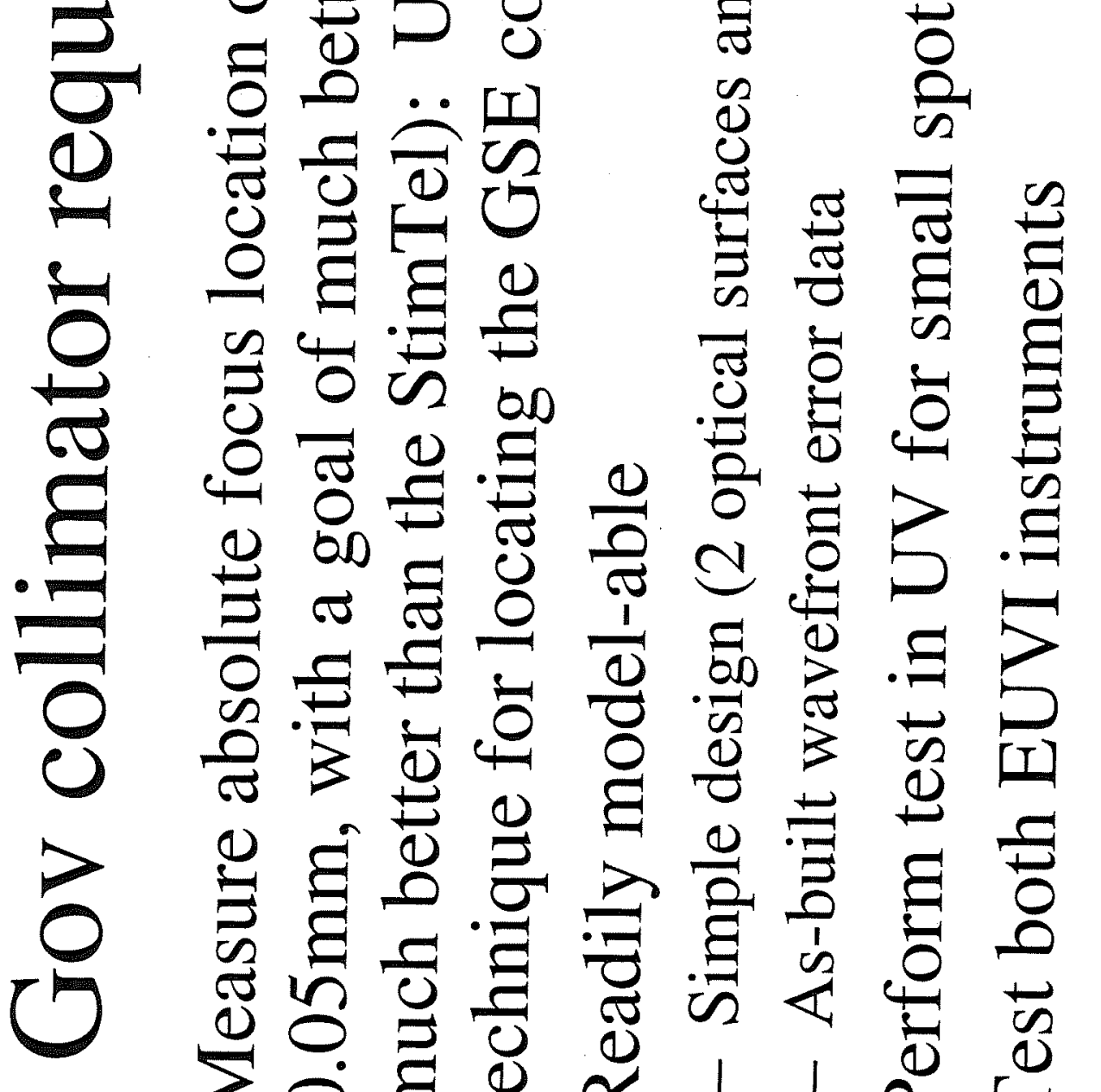

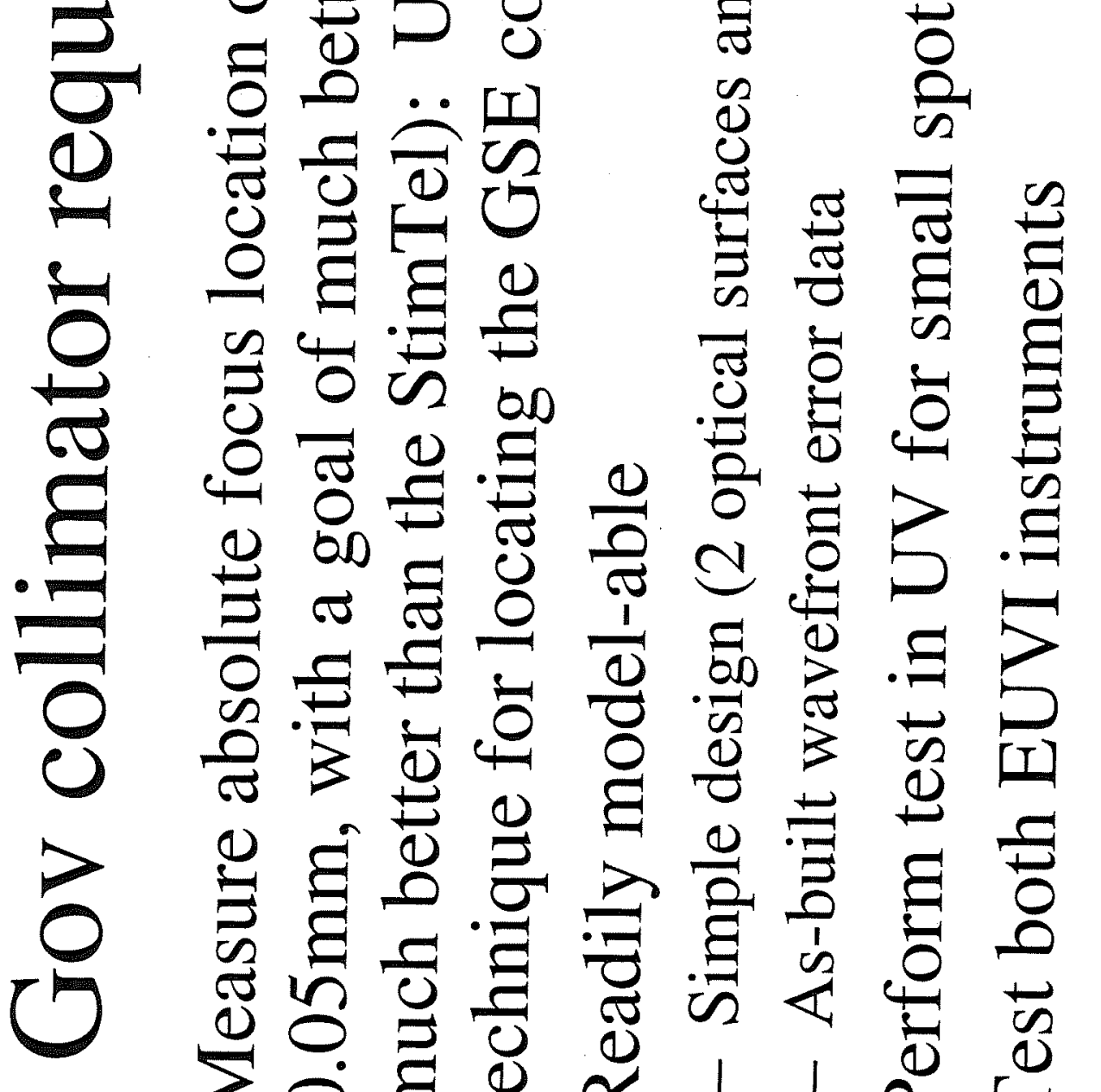

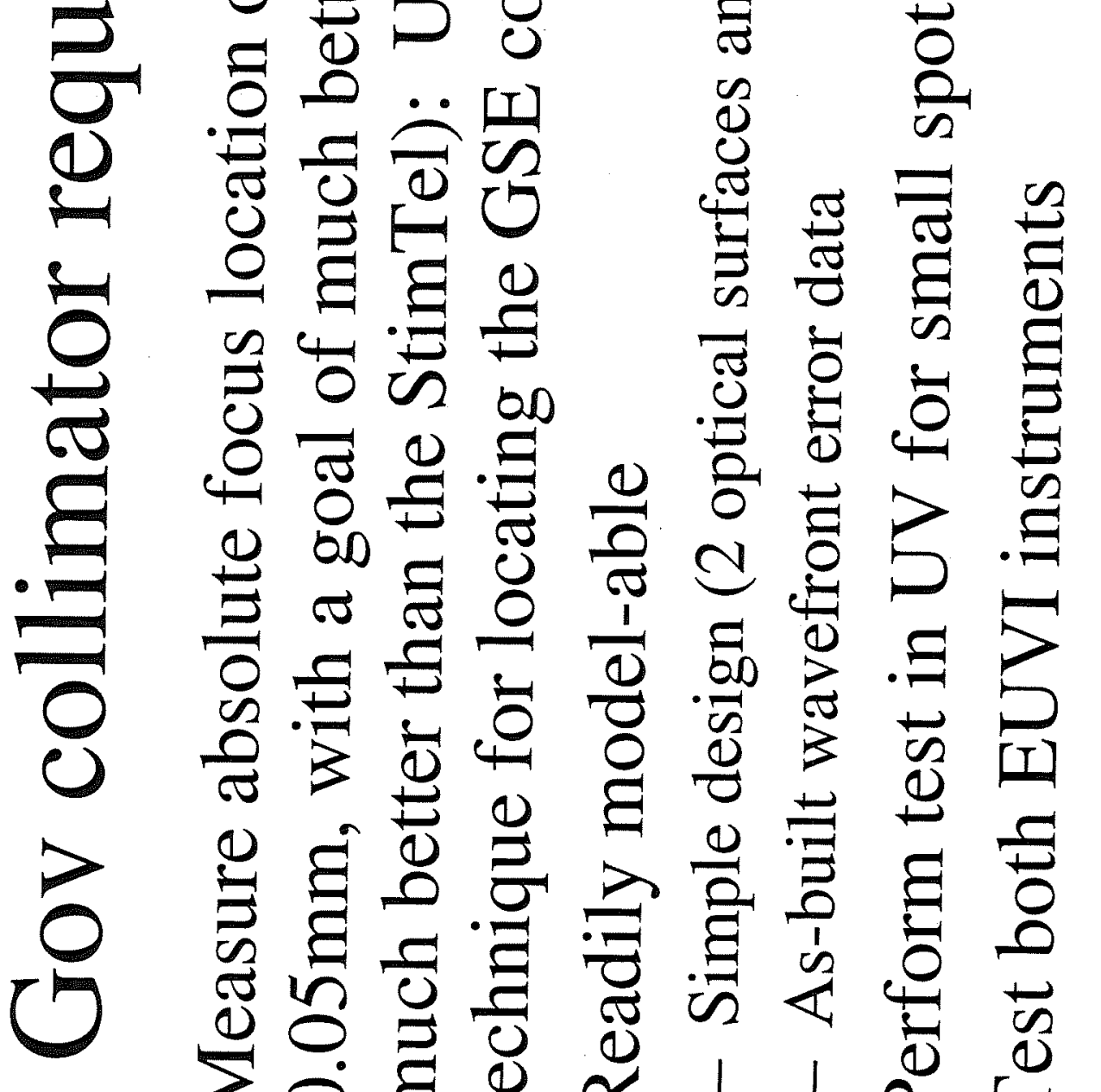

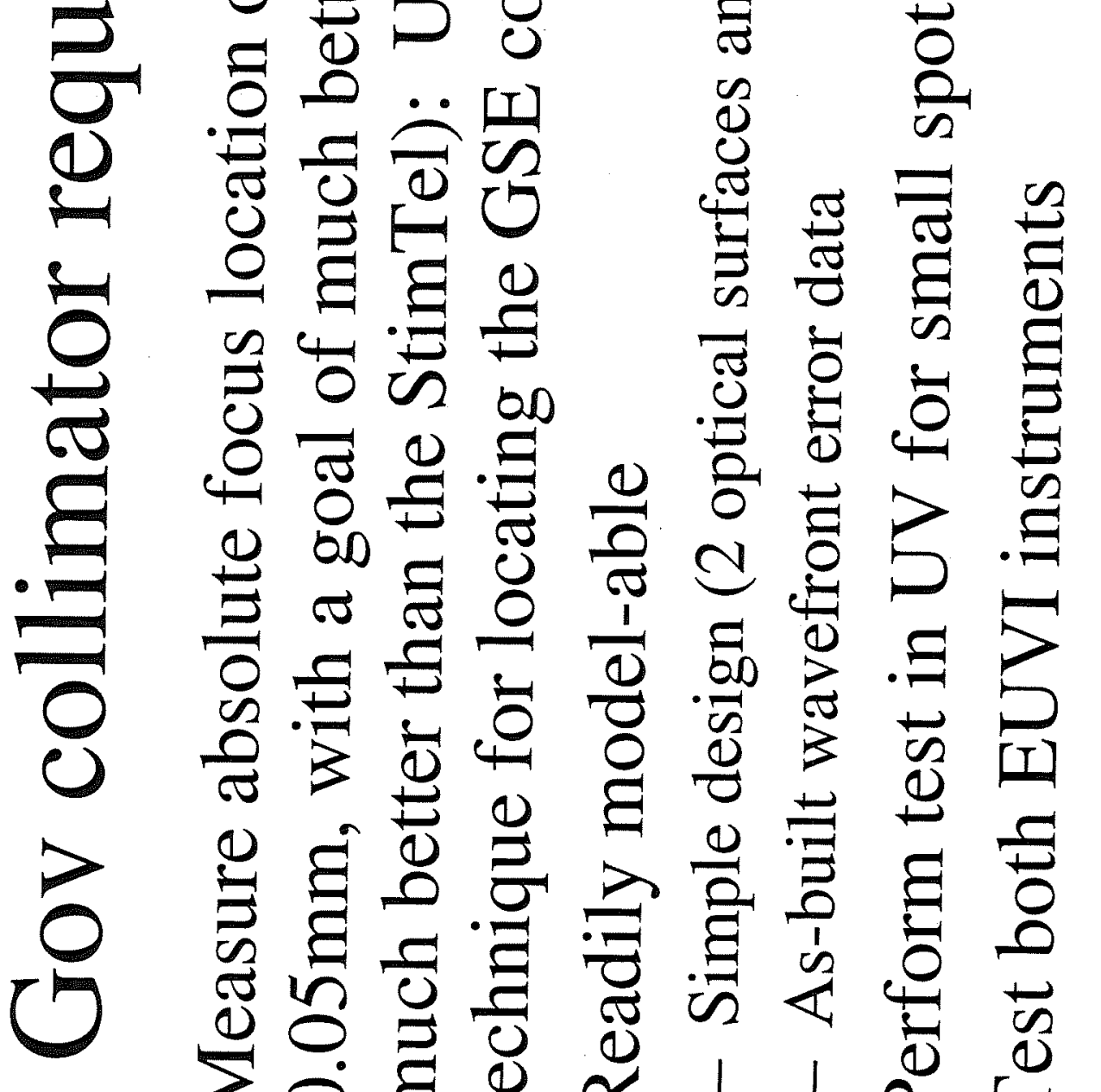

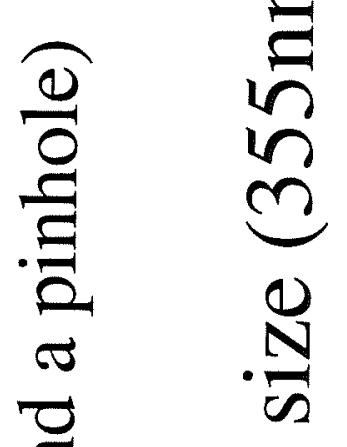

De

$\sigma_{n}^{4}$

$\infty$
$\dot{2}$
5
0
0
0
0
0
0

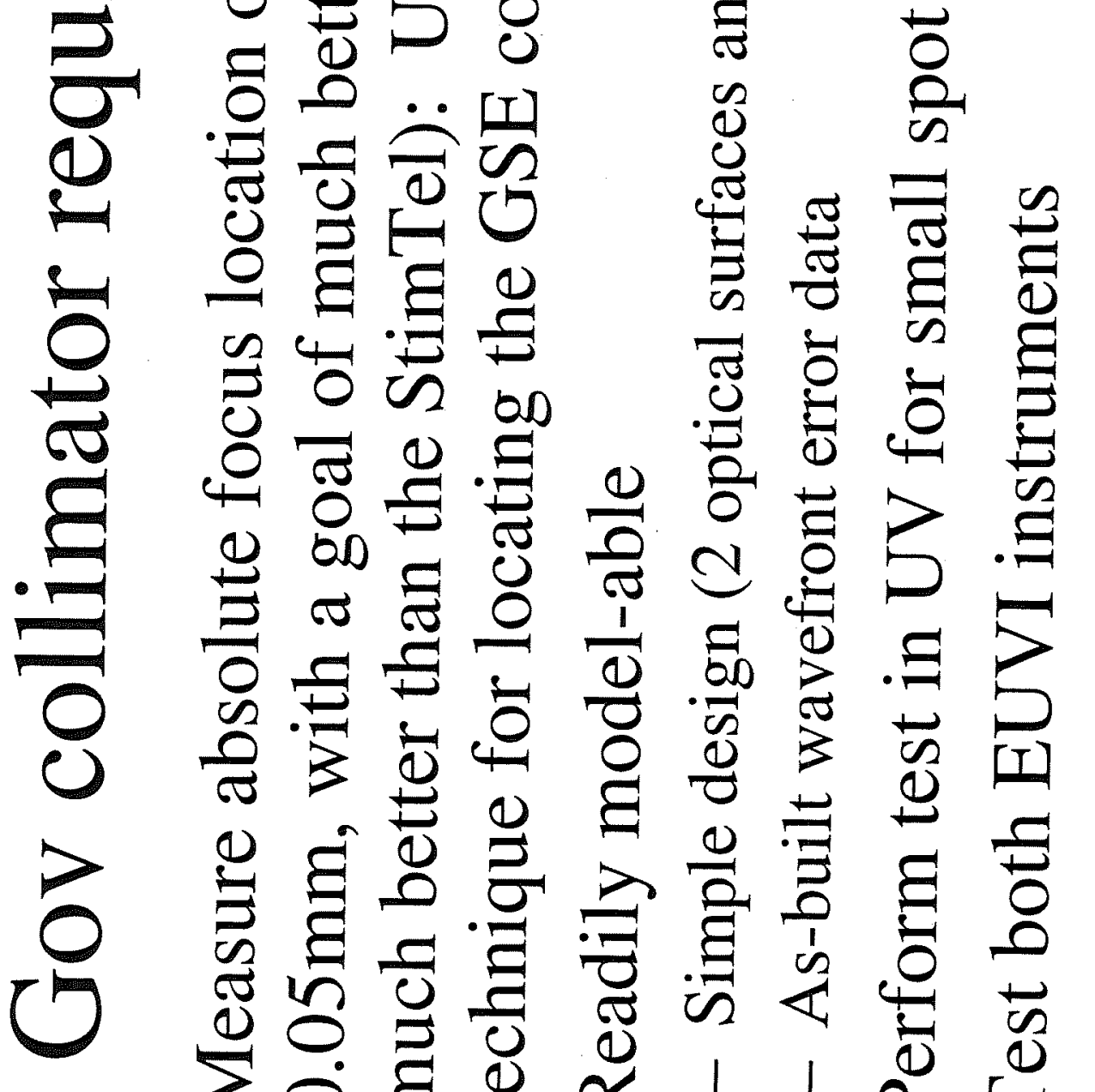

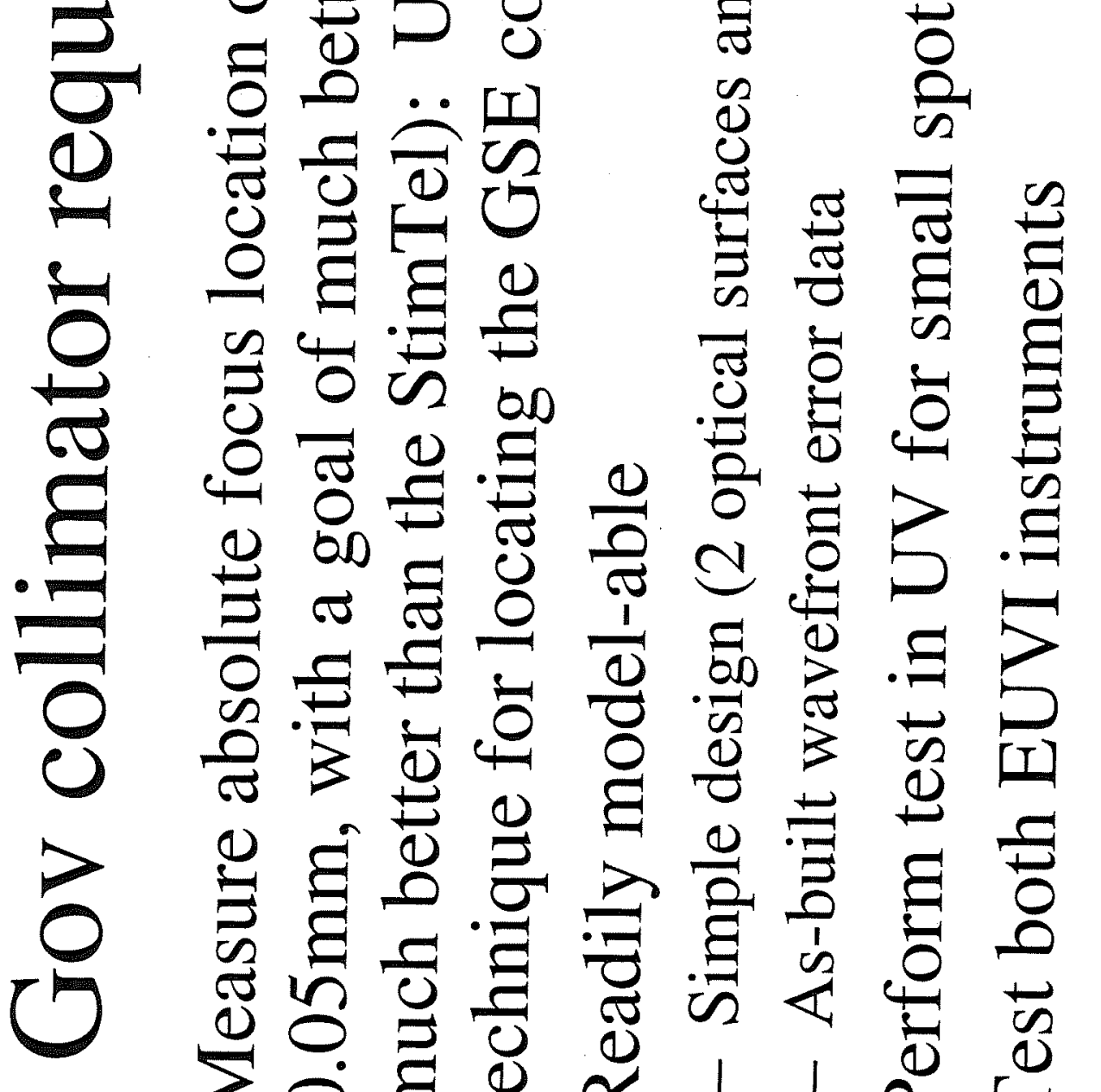

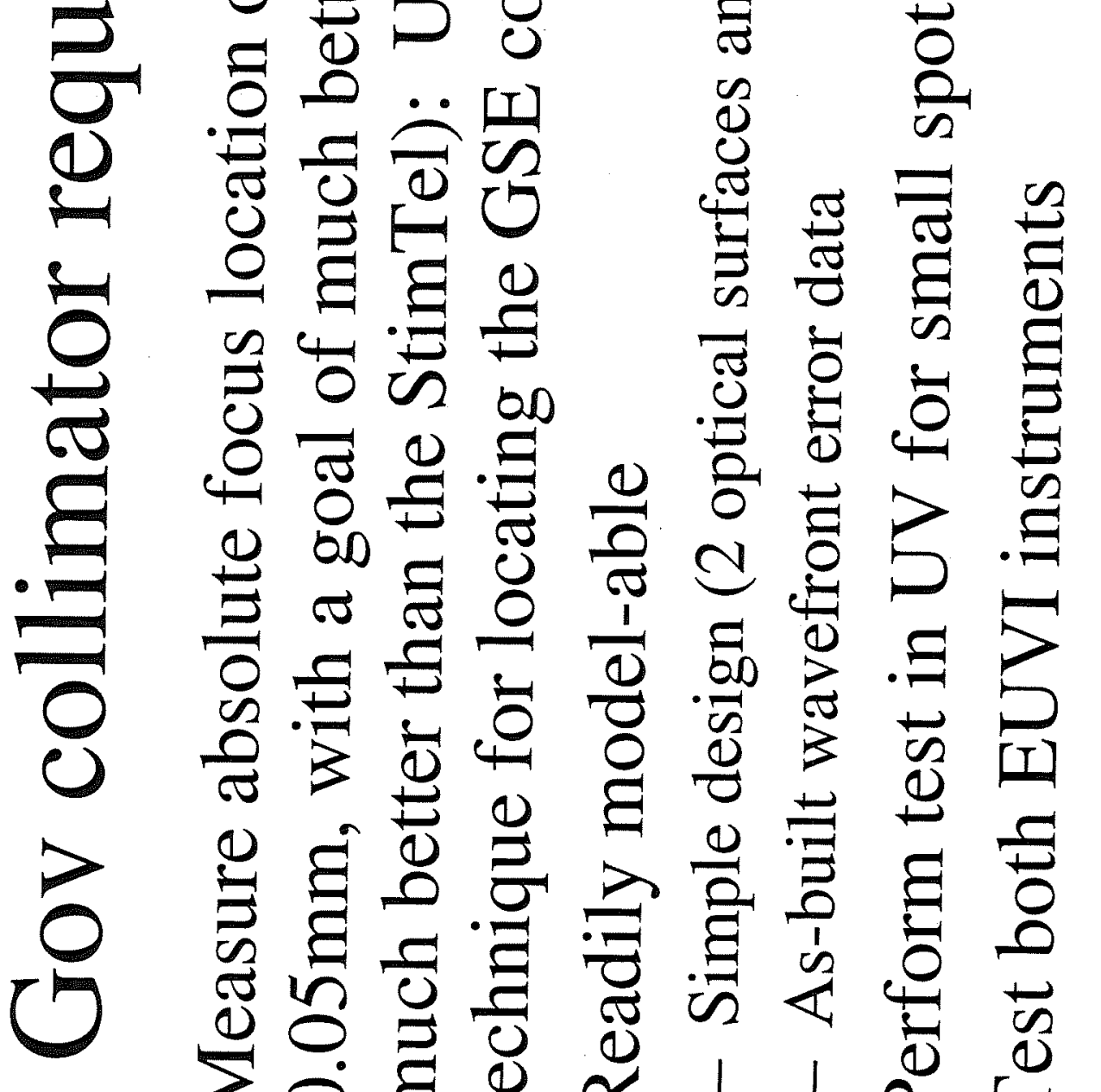

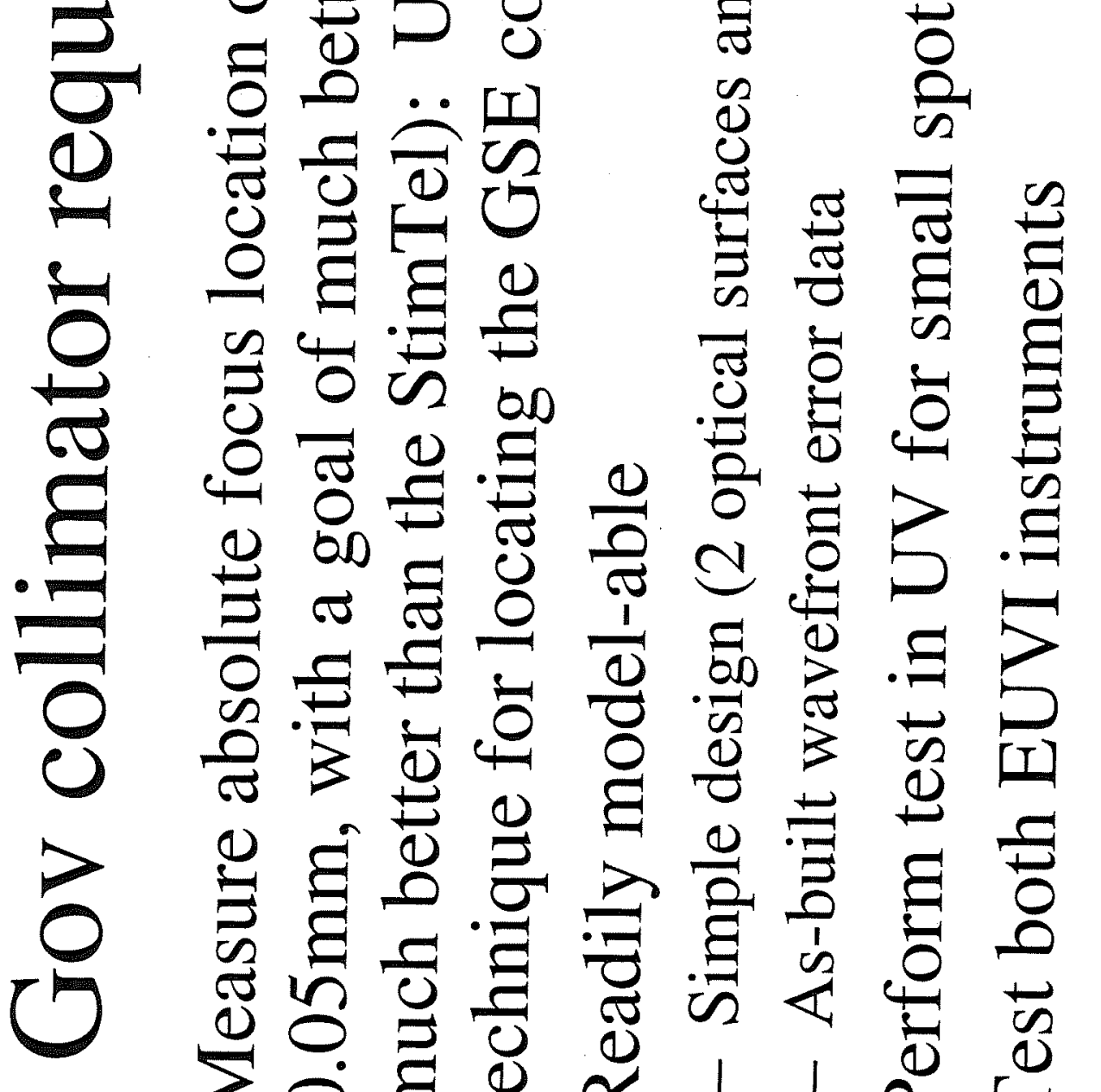

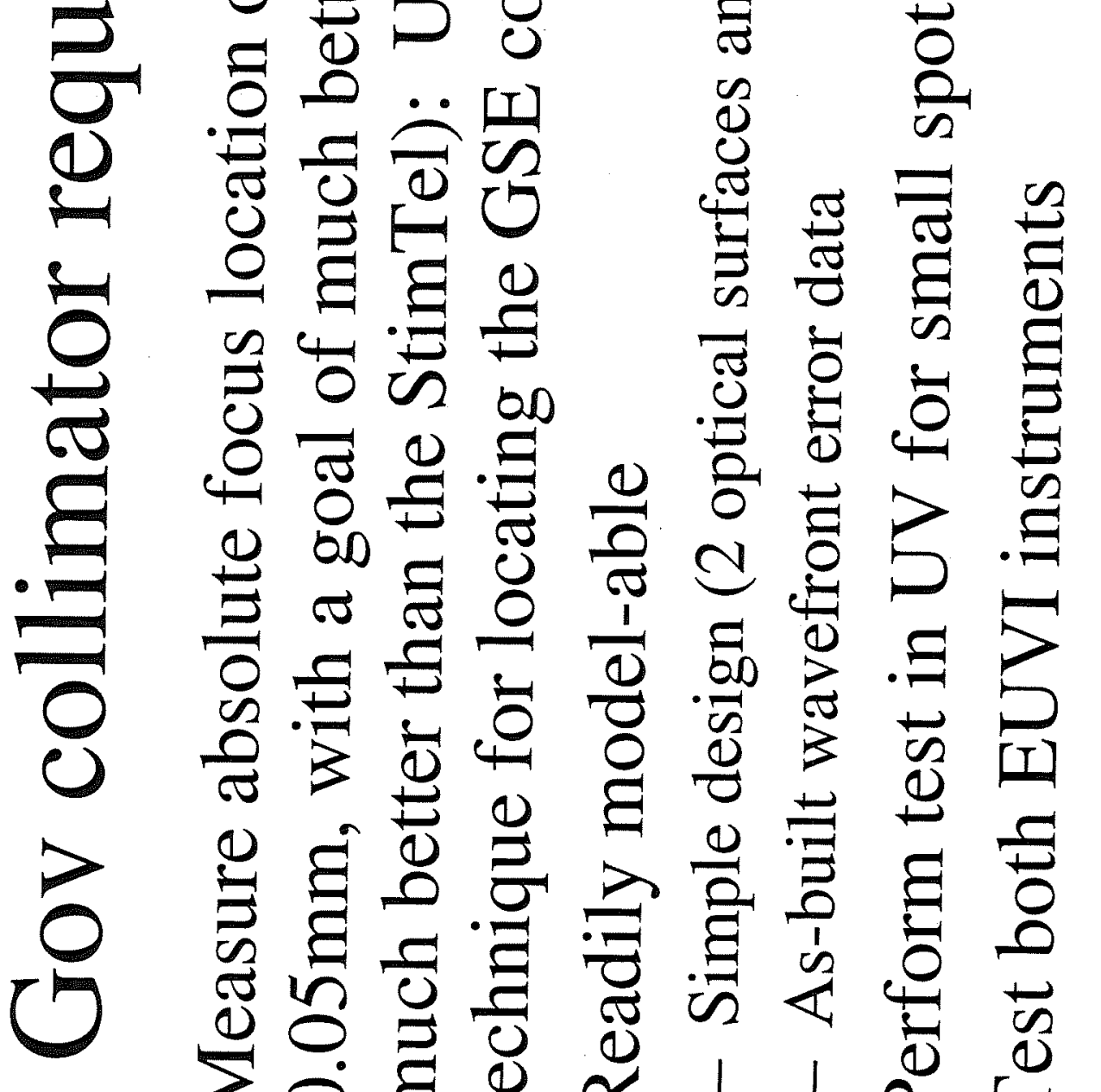

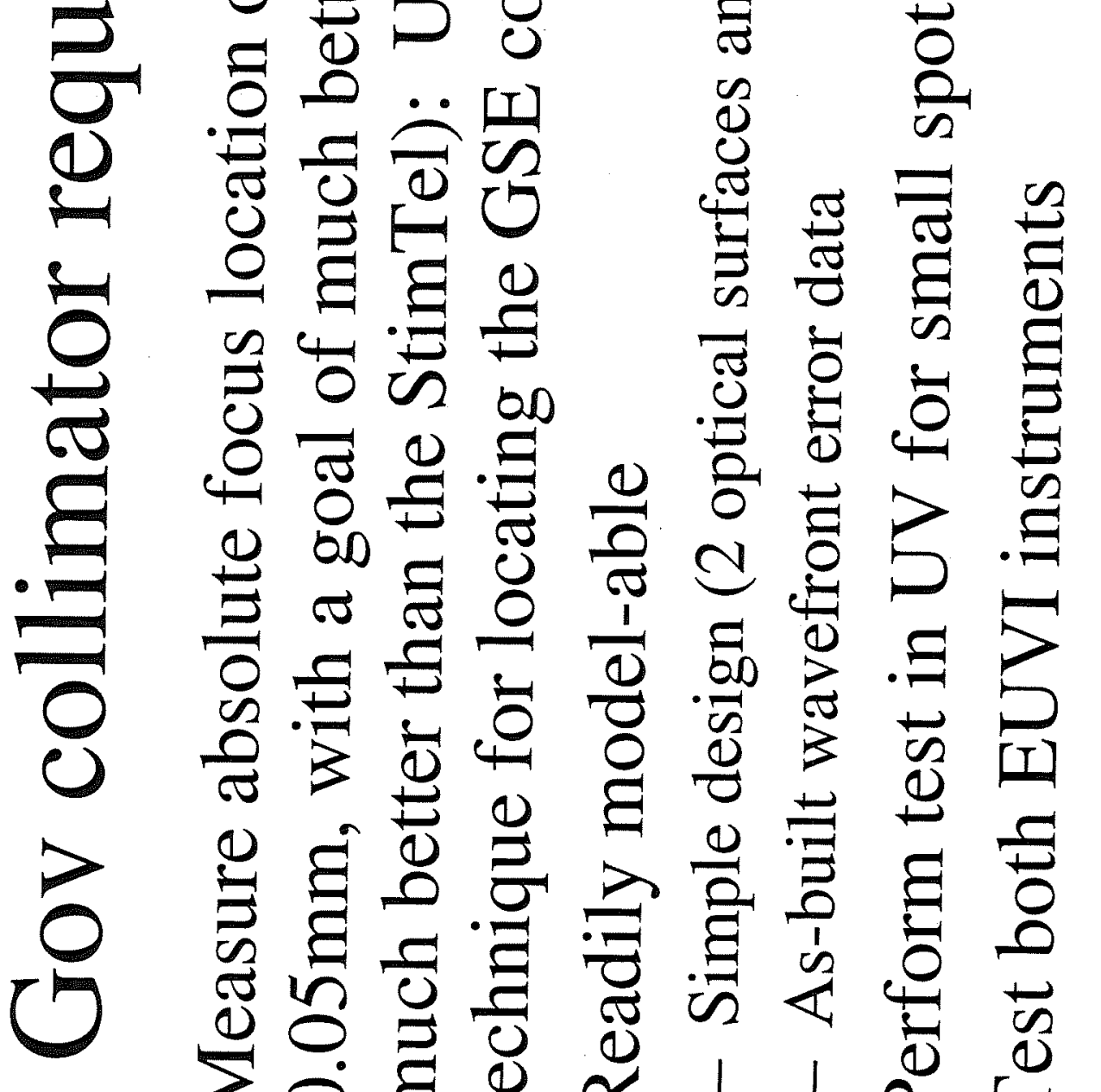

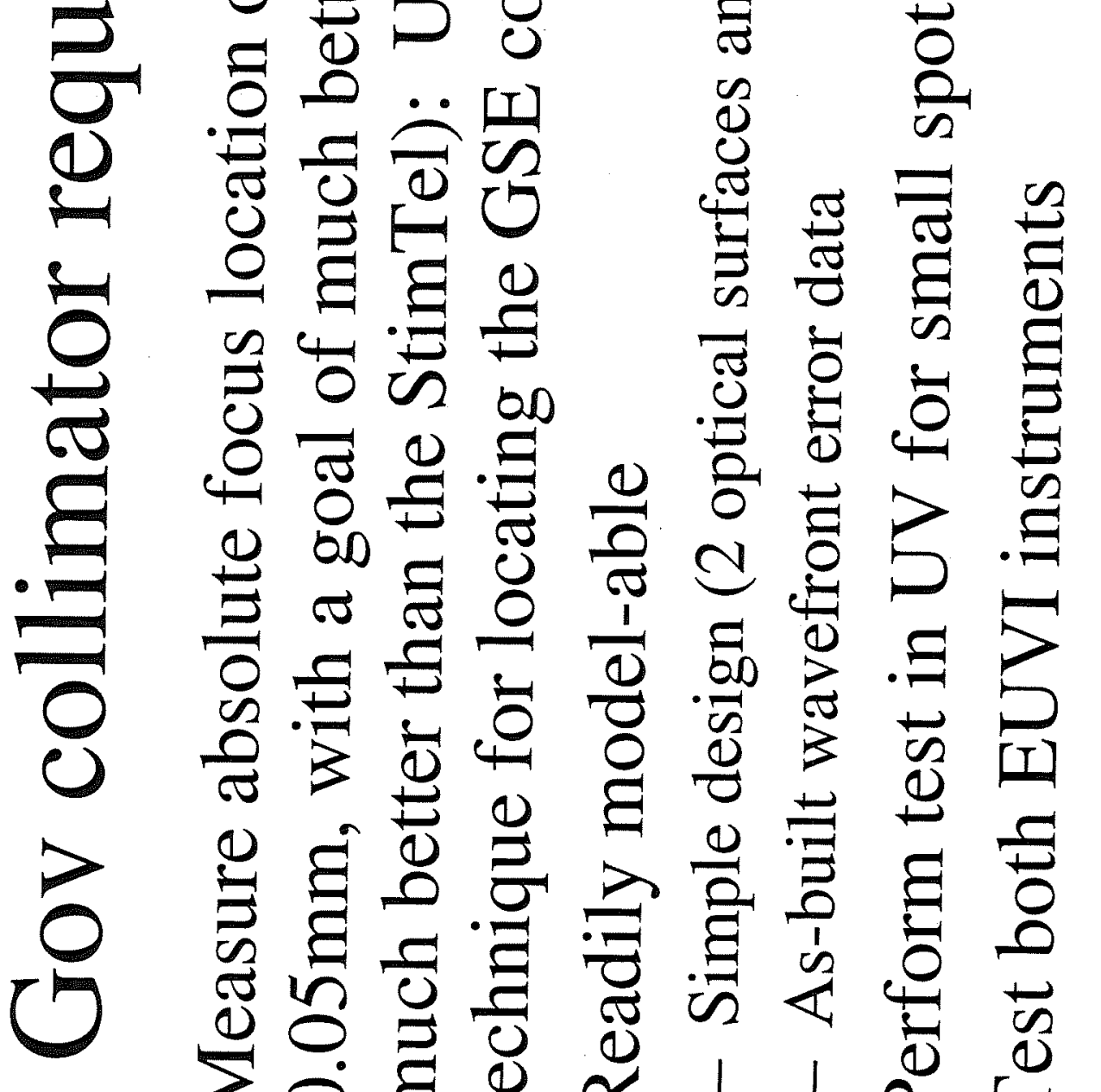

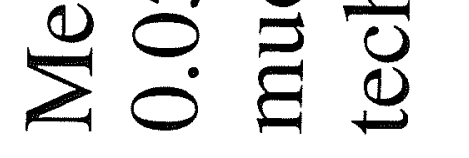

(i)

苞

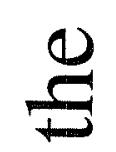

E

$\otimes$

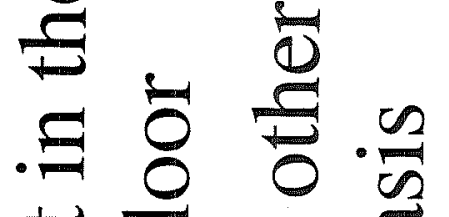

$+\underset{\sigma}{\square}$

i 0 i 0

(1) $\$ 0$

040

$\frac{2}{0} 0 \div$

了.

$\pm n \approx 0$

$\Xi>0 \Xi$.

कर

प्र 5 ष

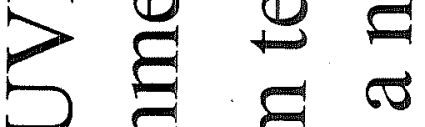

(I) 0 ह

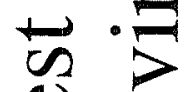

$+0$

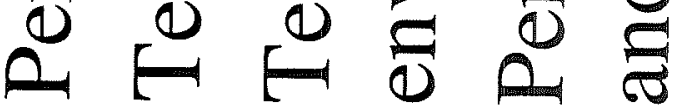



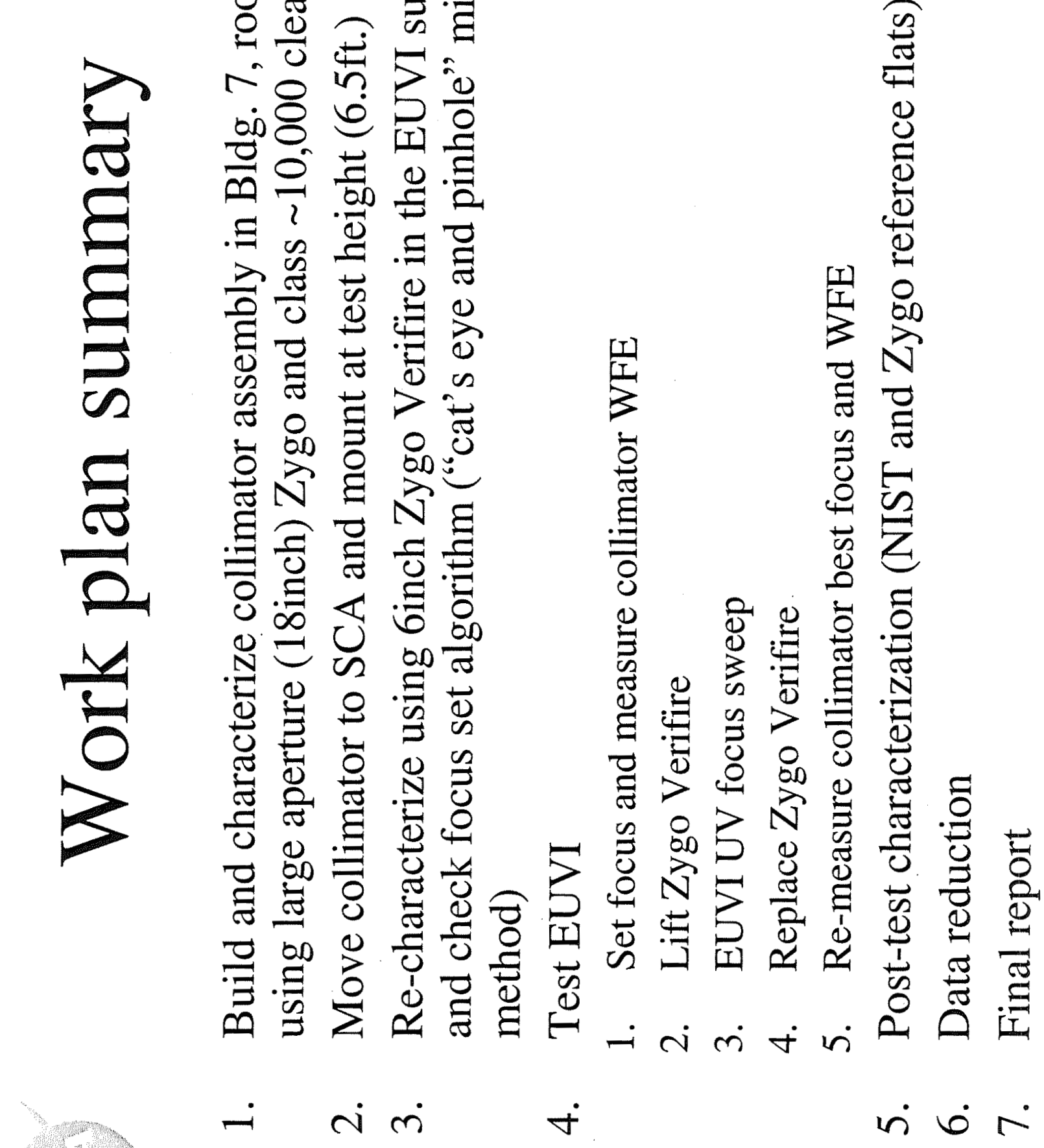

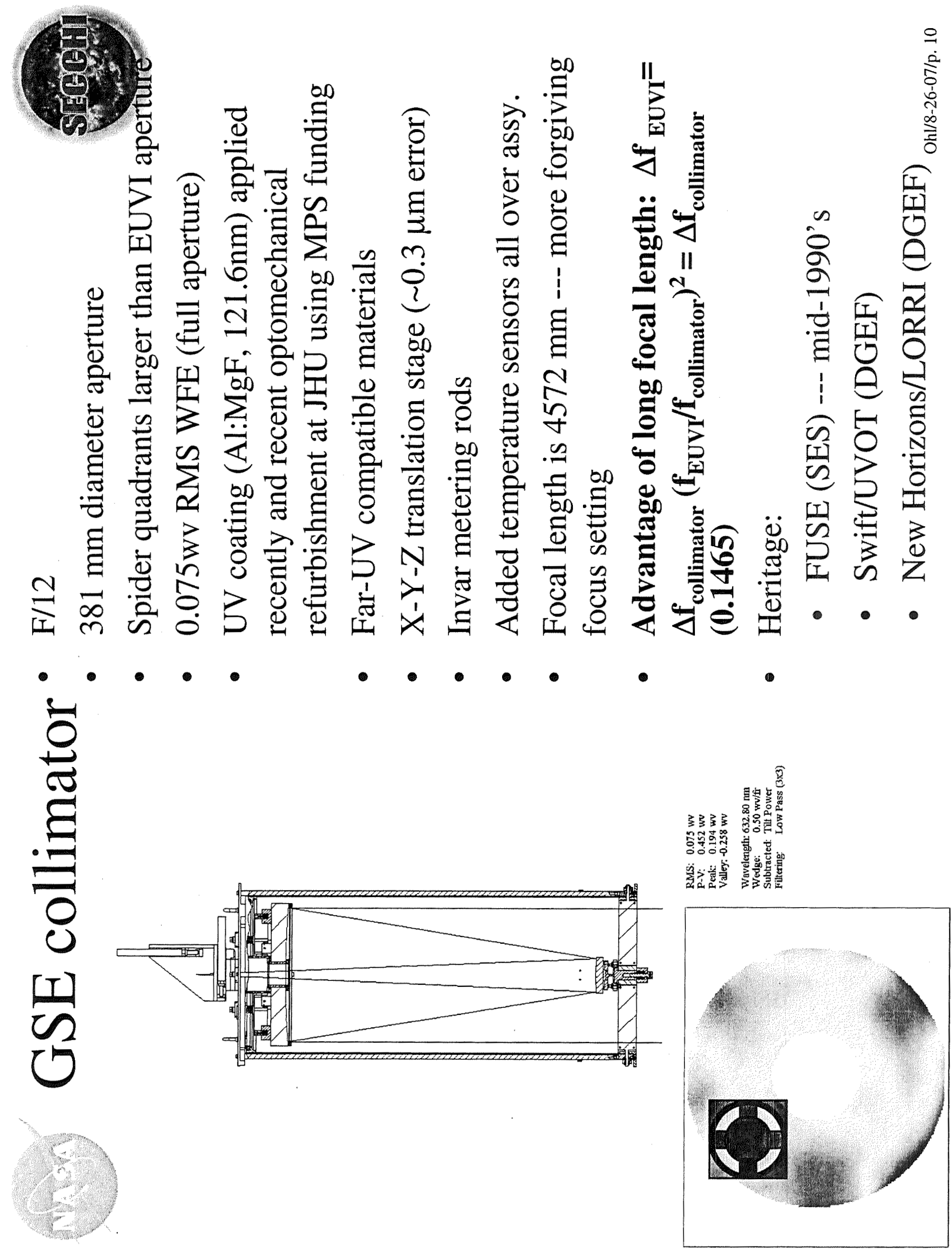


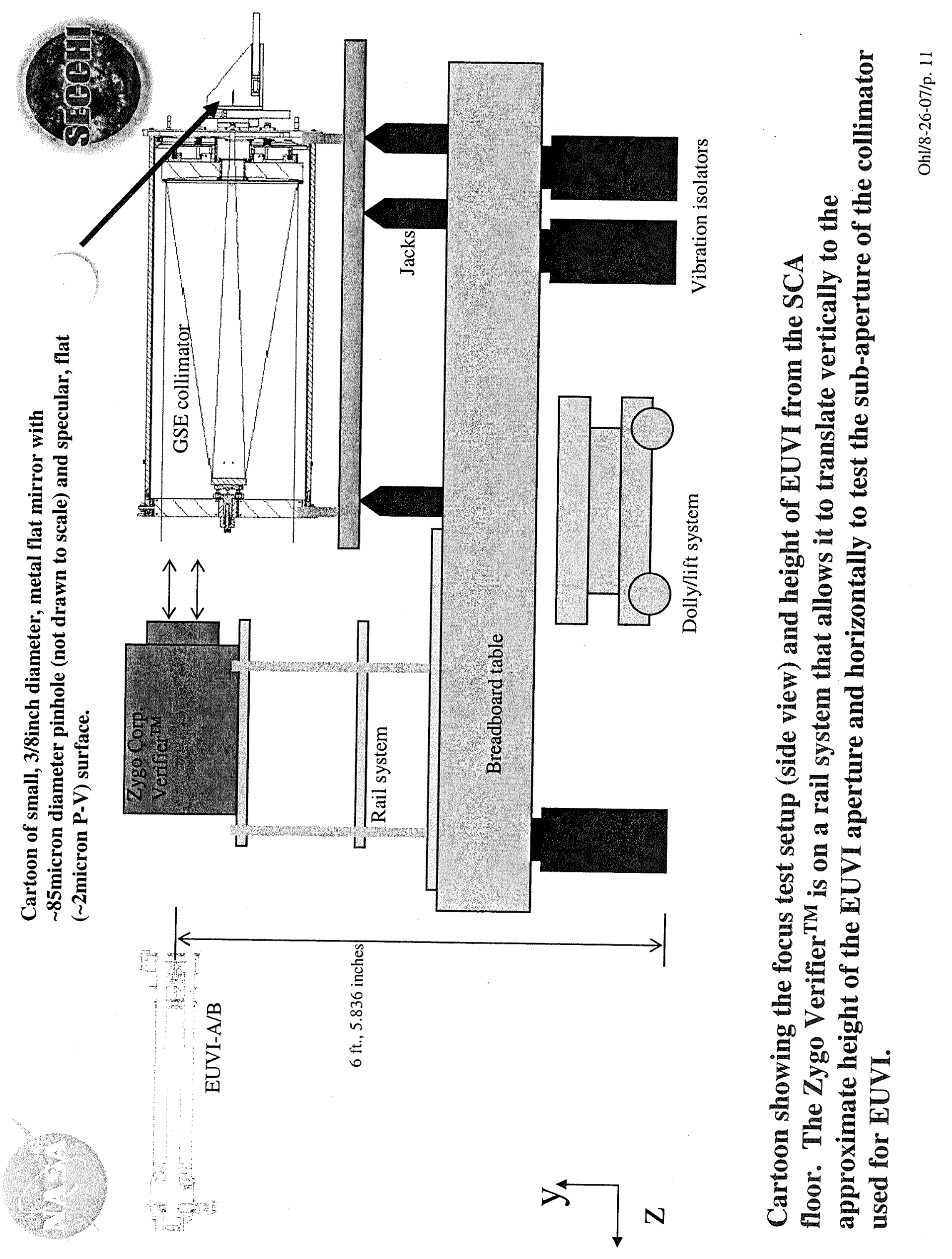




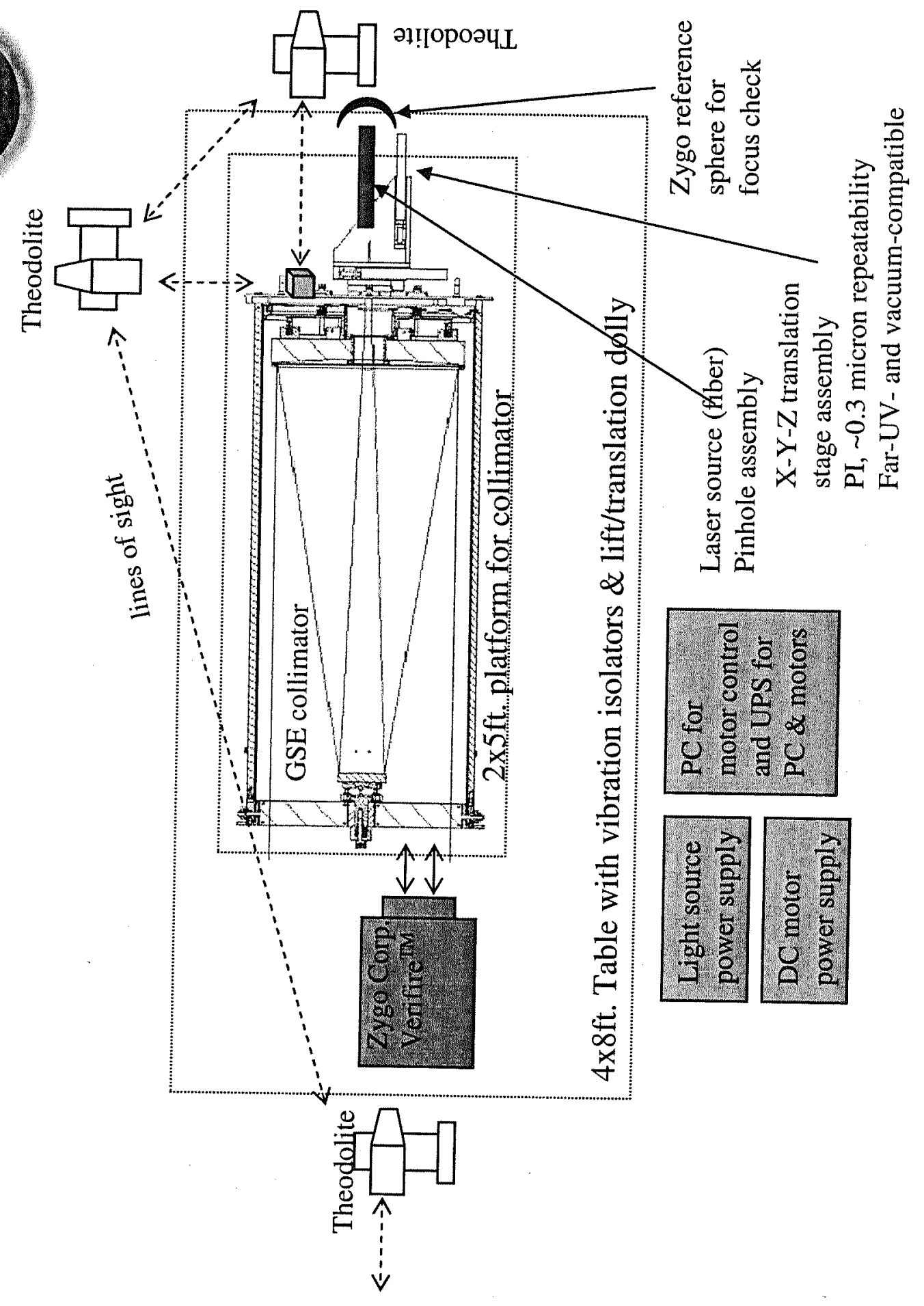

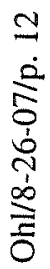

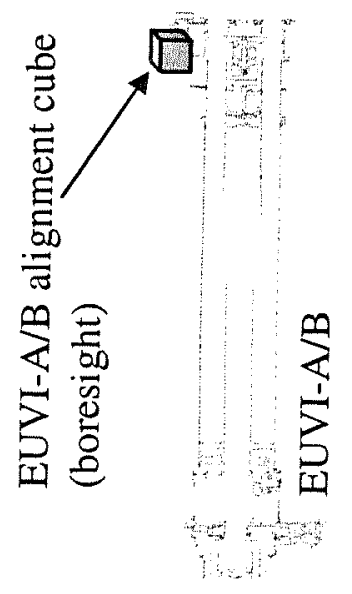

䓪 


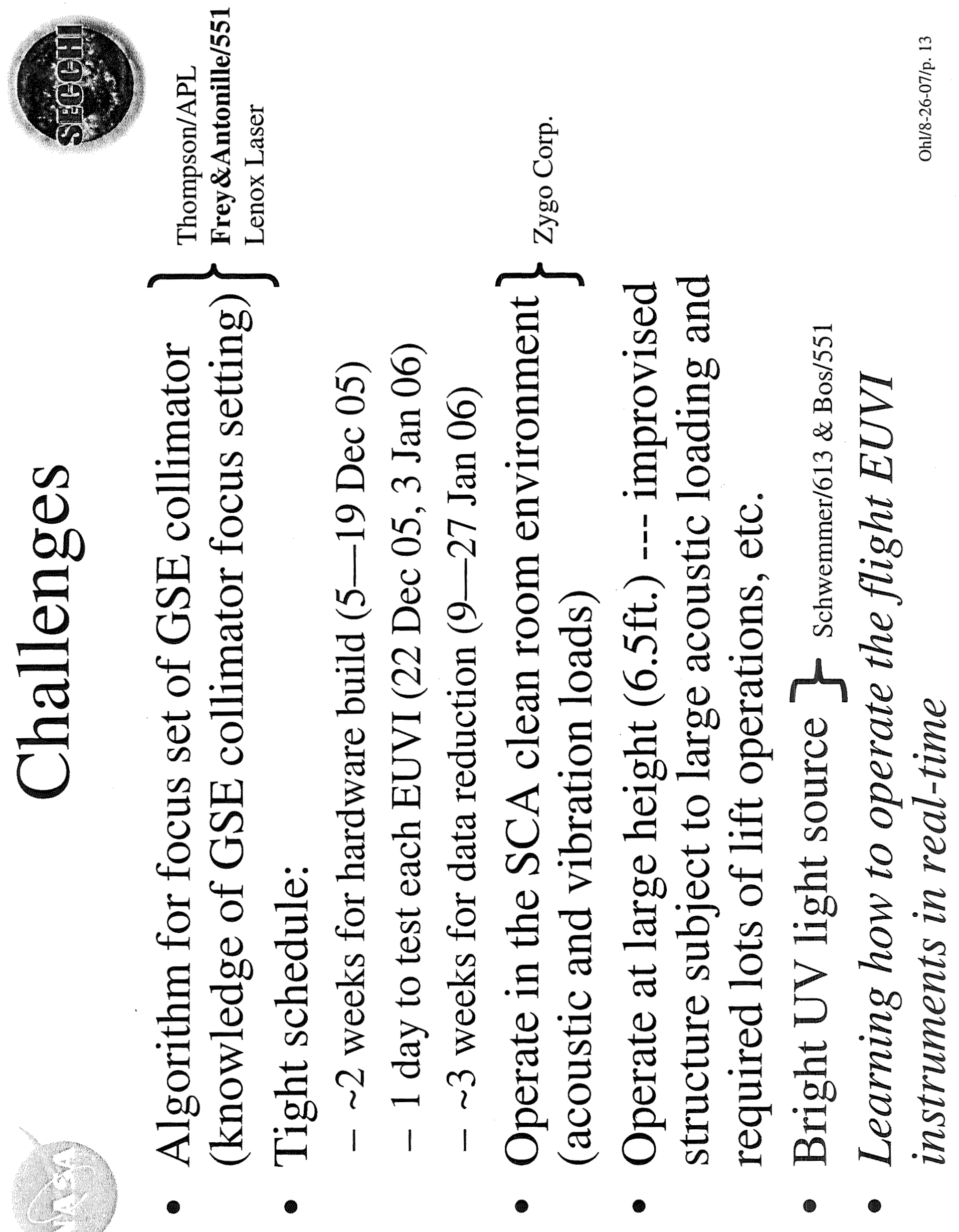



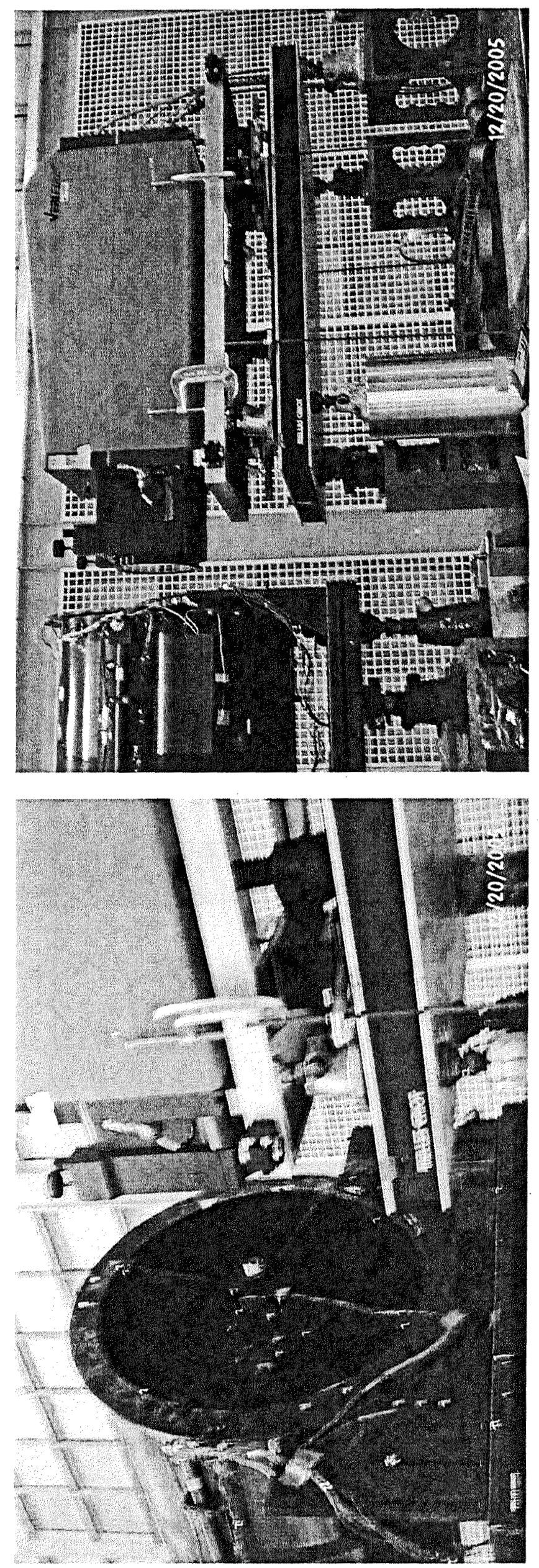
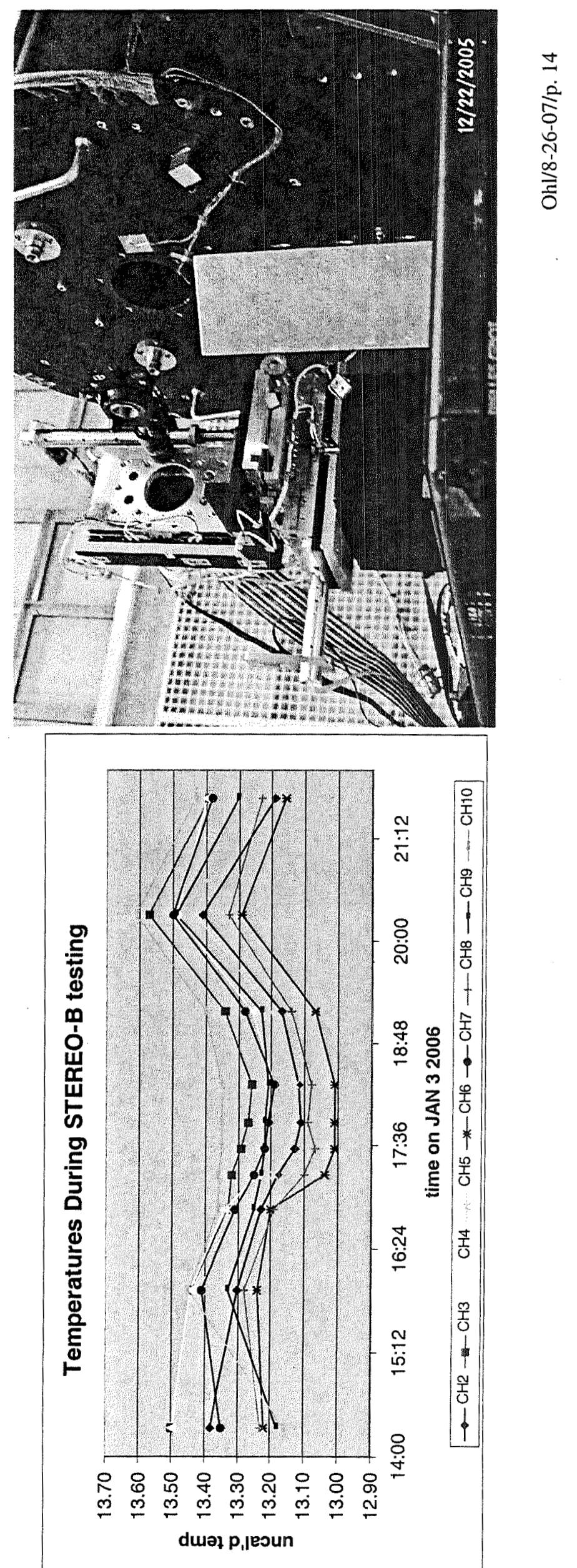

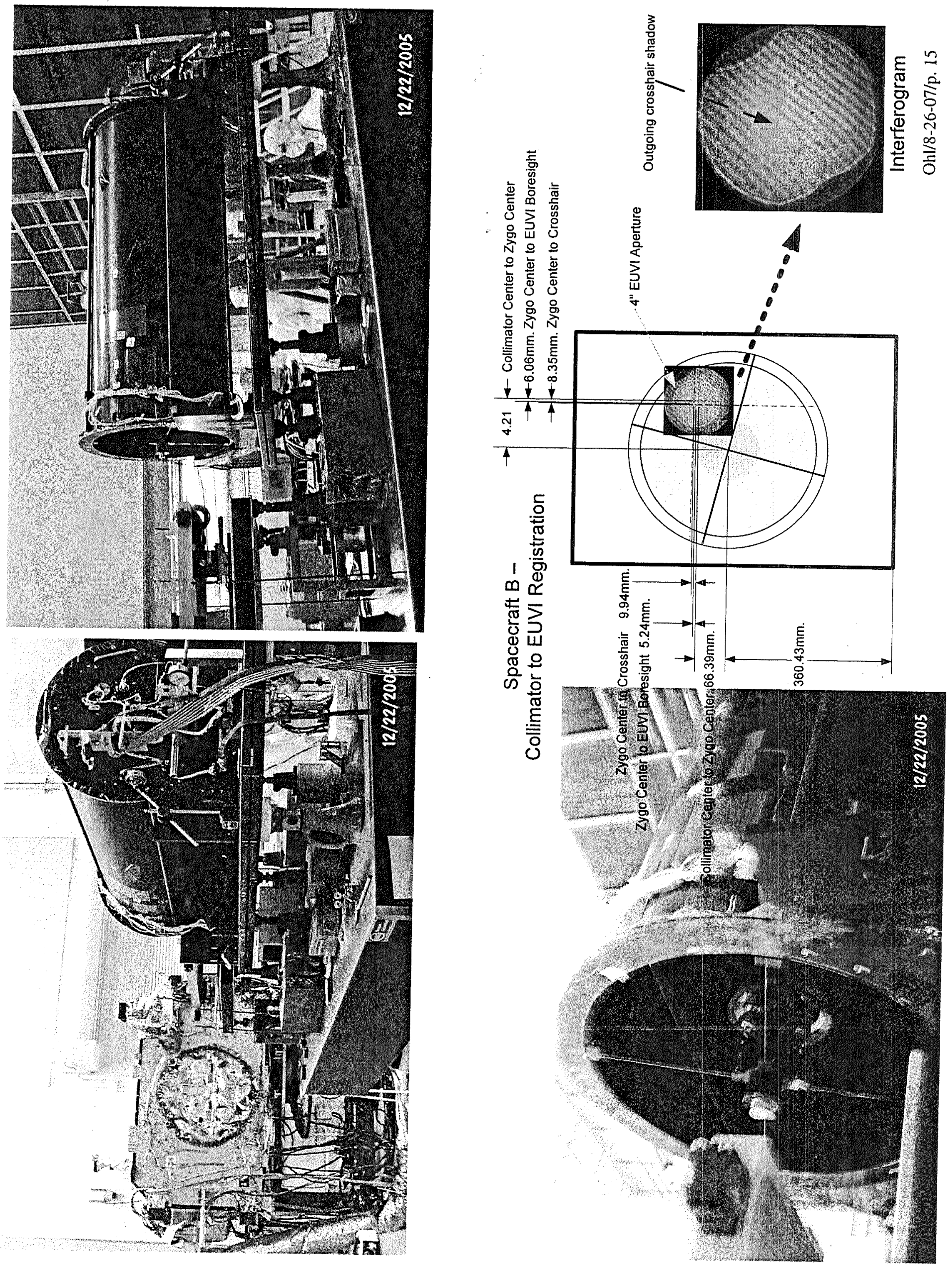
bo

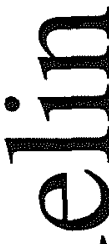

8

0

$\varepsilon$

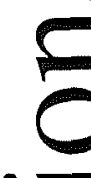

-

e

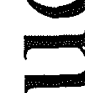

8

(1)

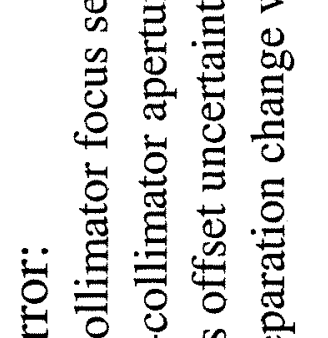

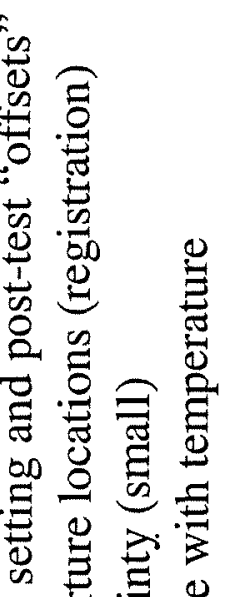

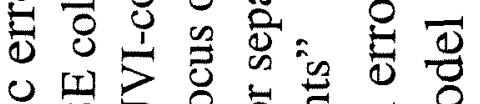

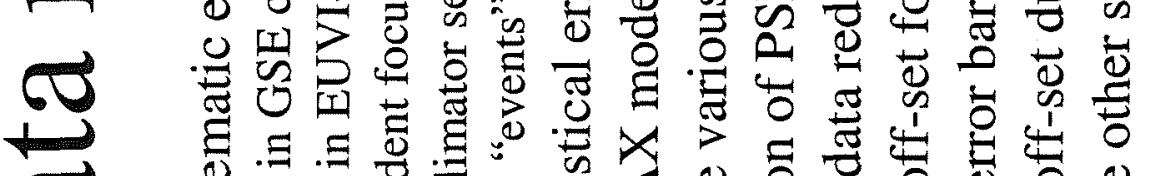

б

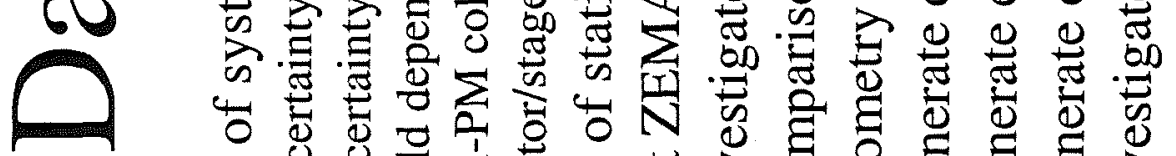

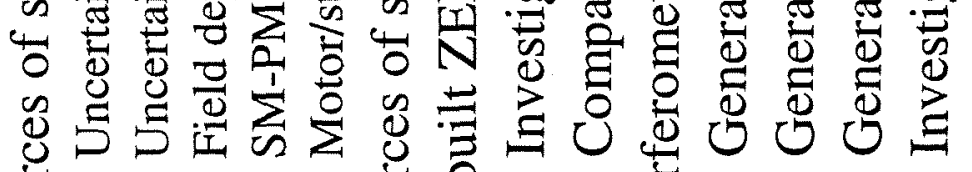

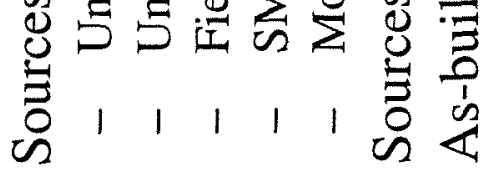
$\stackrel{\square}{\Xi}$

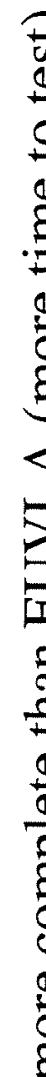

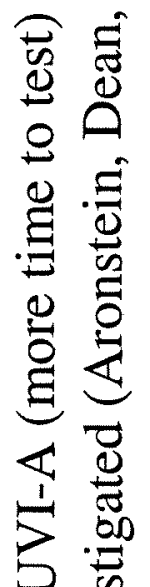

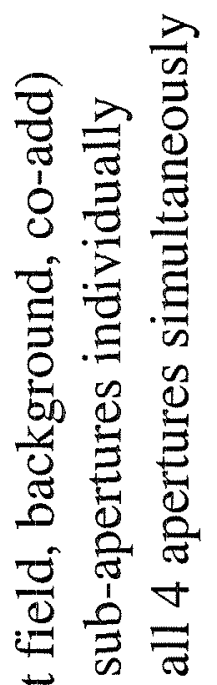
记 (1) 急 吾

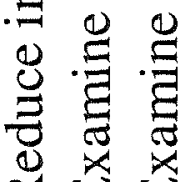

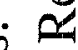
运 


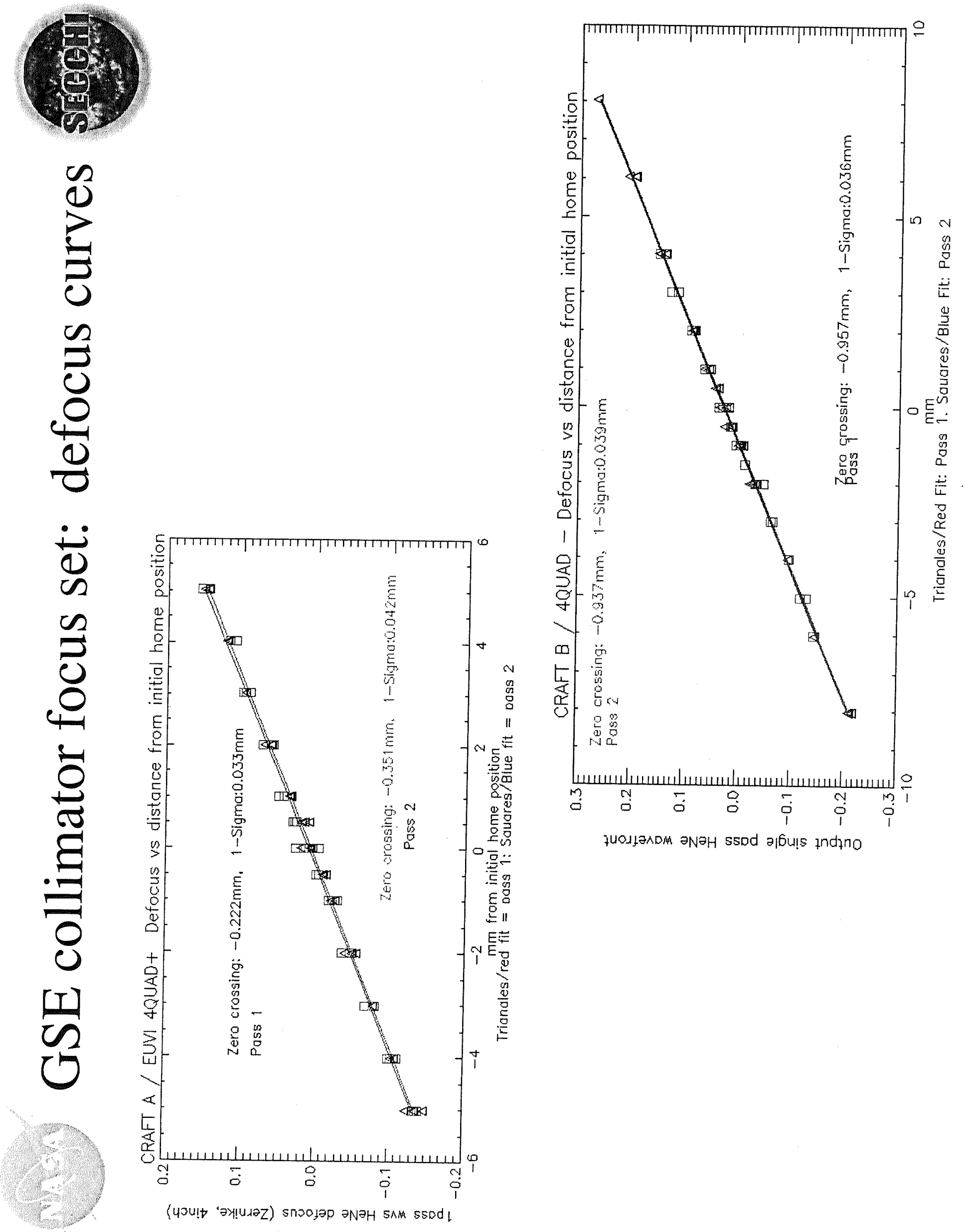



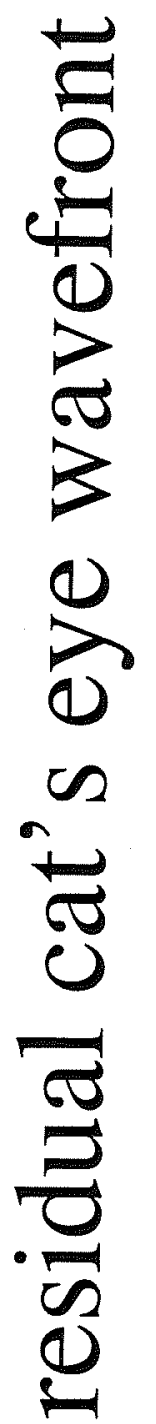

(1)

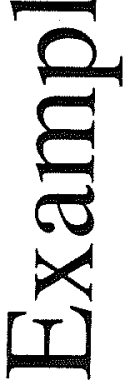

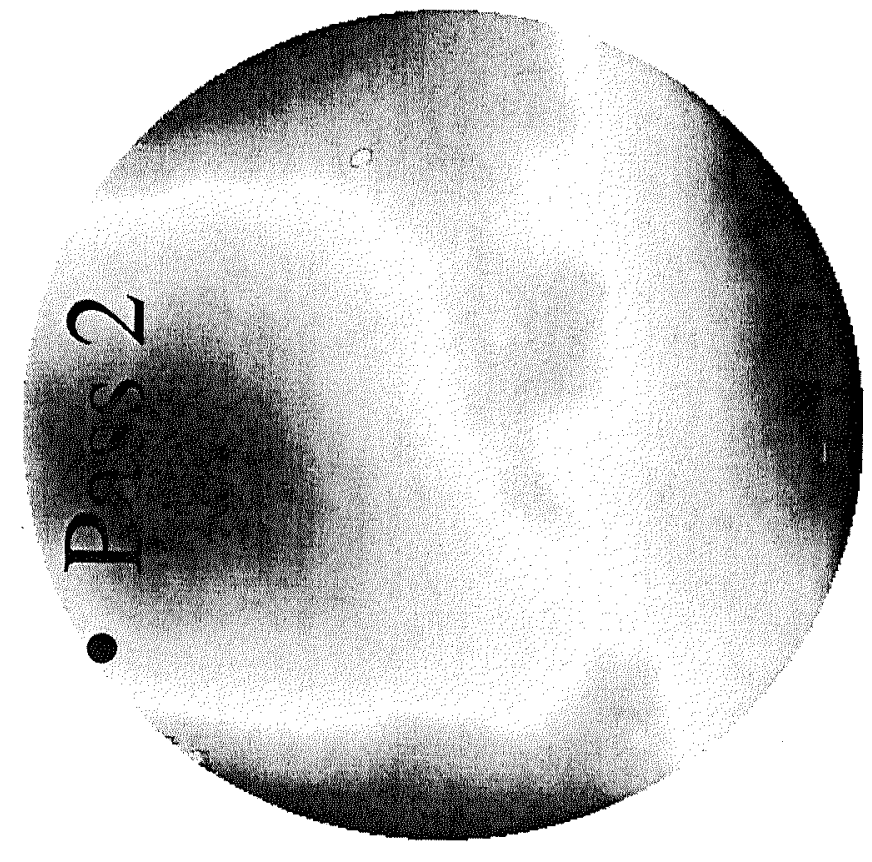
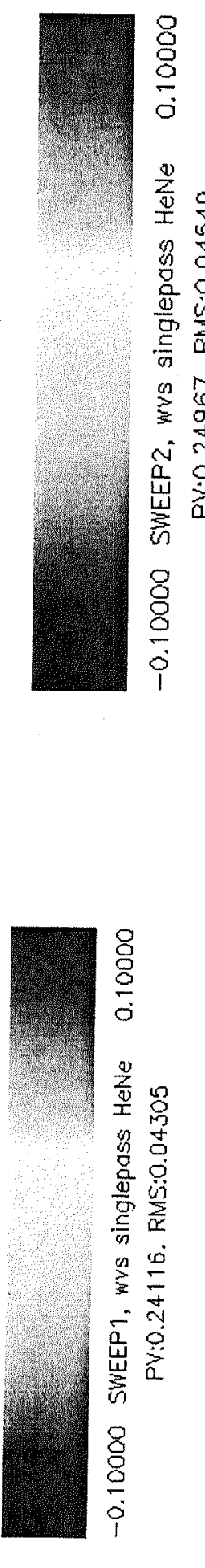

o $\quad \cdots$

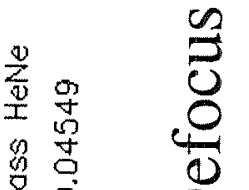

语

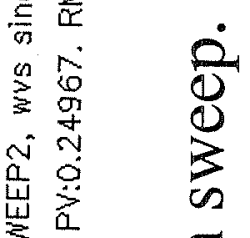
密

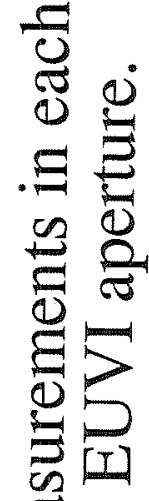

ญे

E

三

$-0$

ฮั

อ ํํㄴ

造:

ह

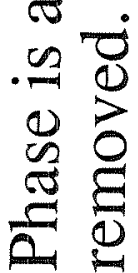




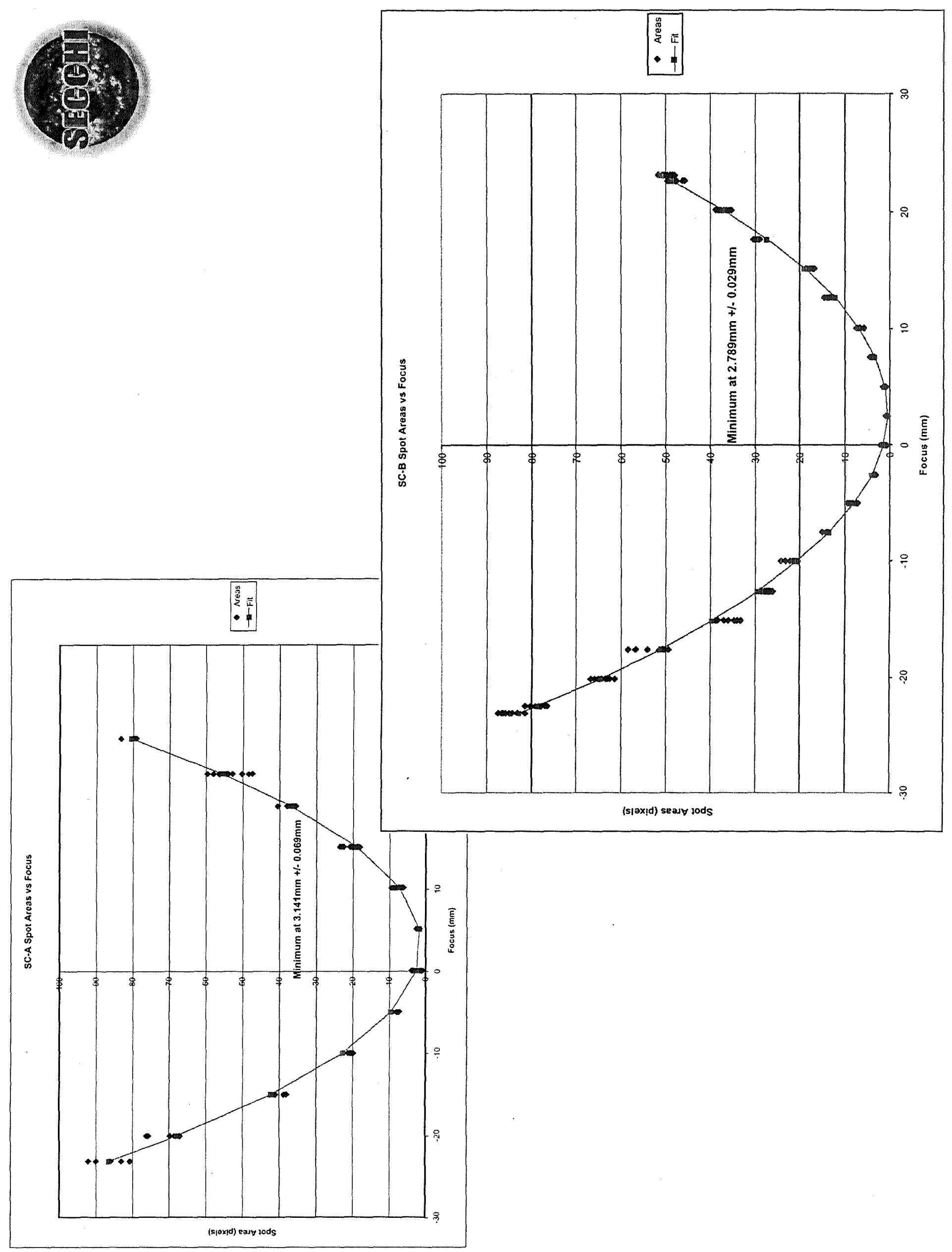




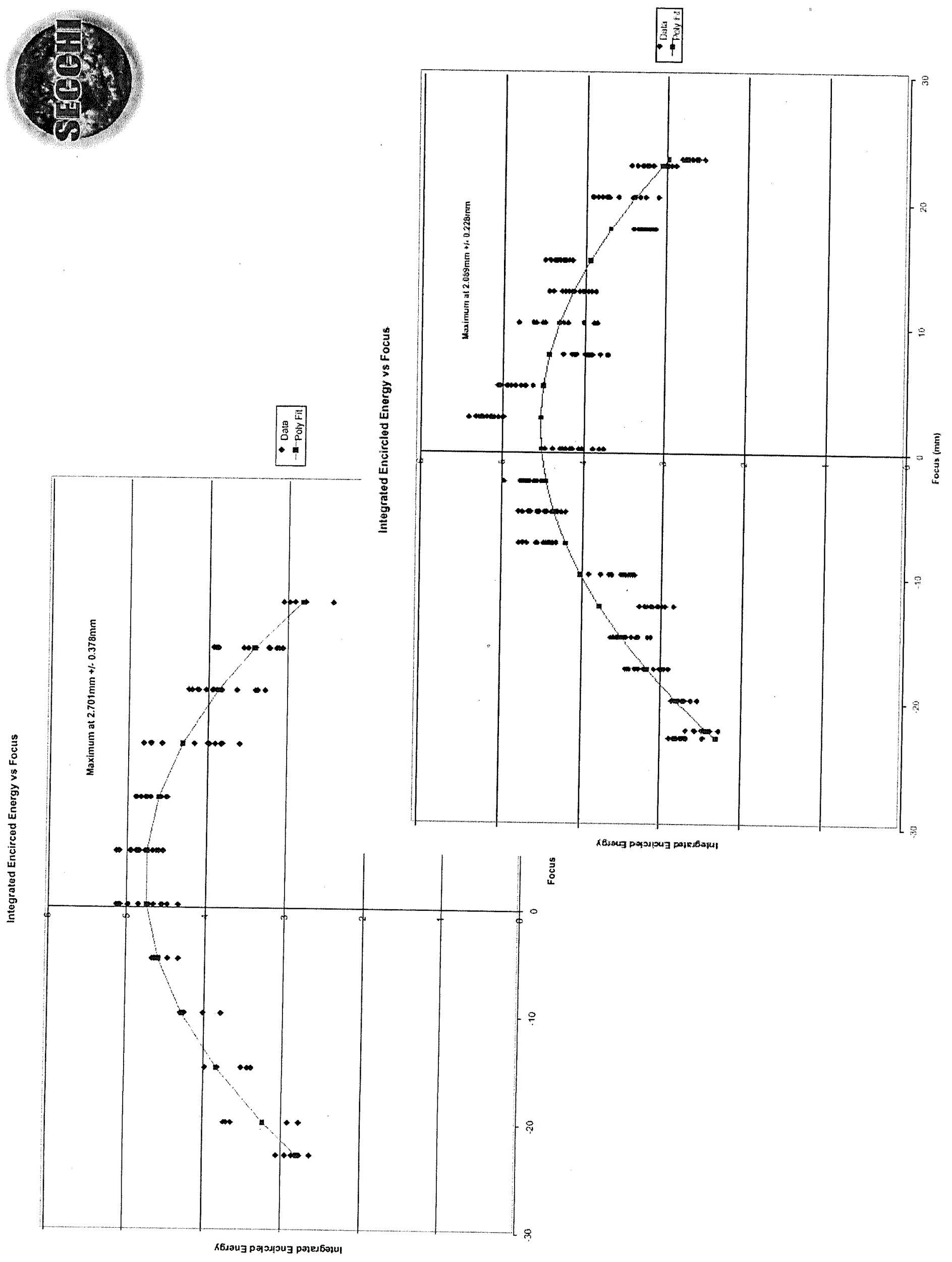




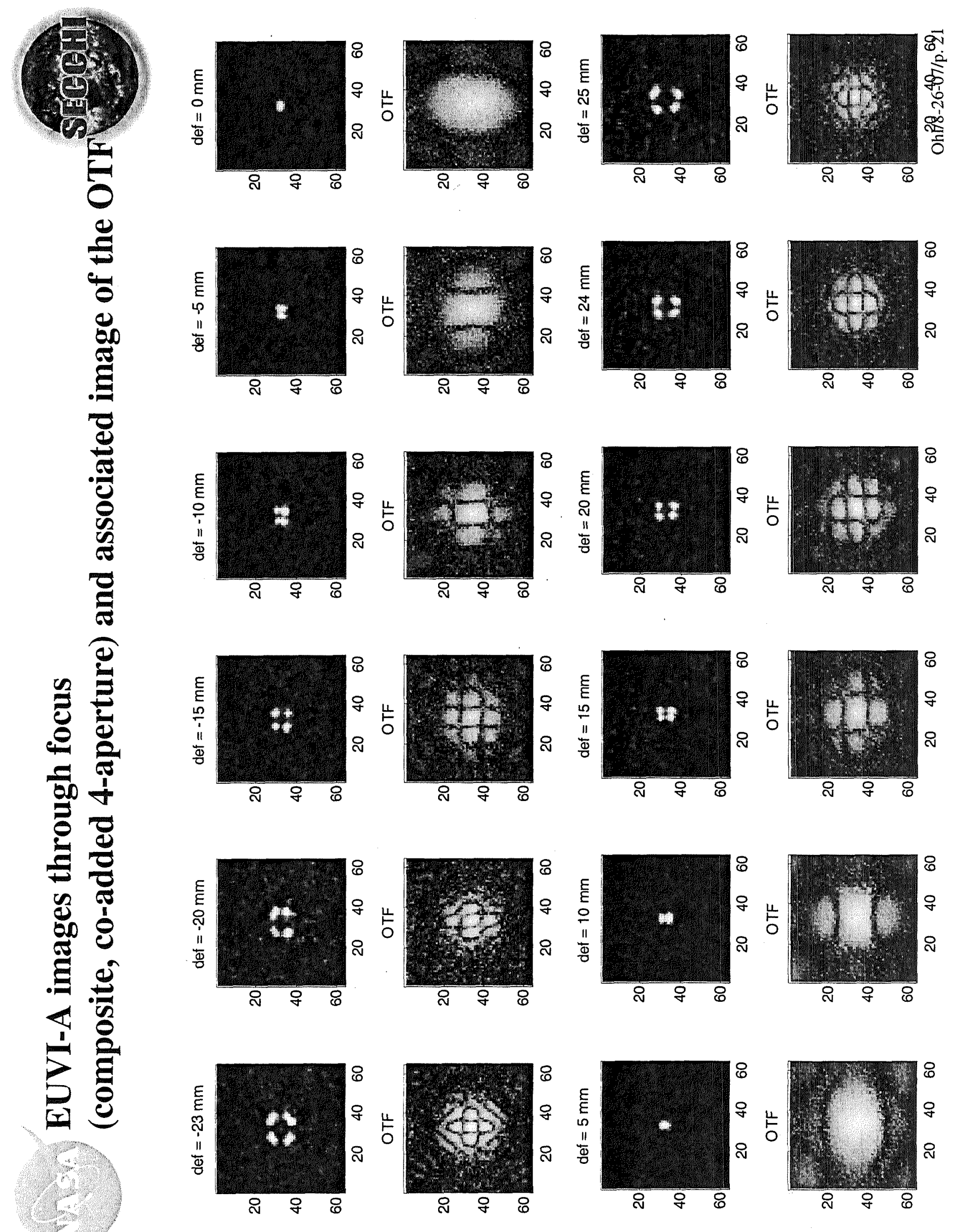




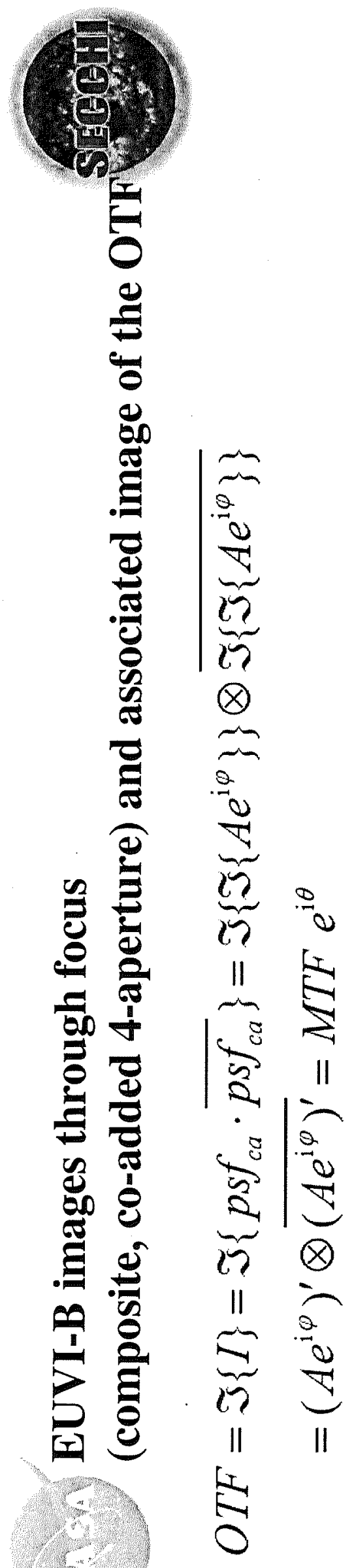

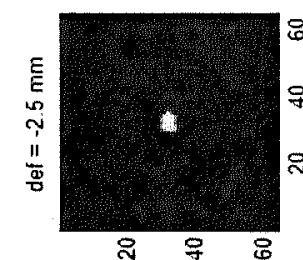

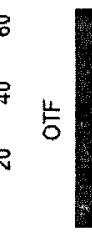
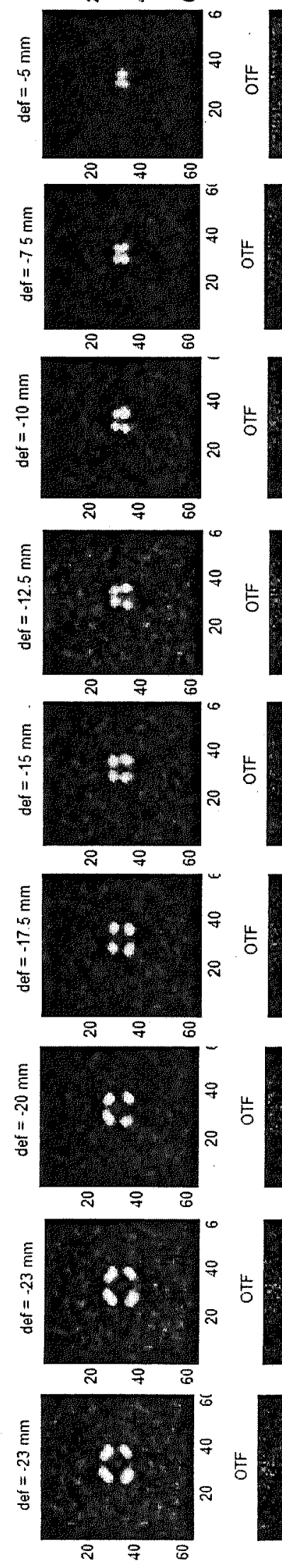
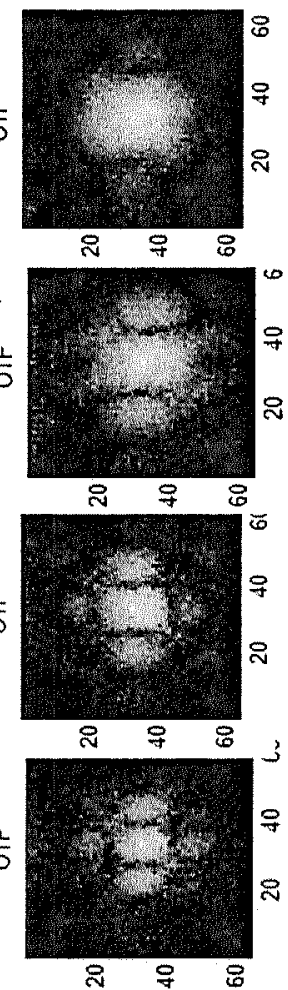

点
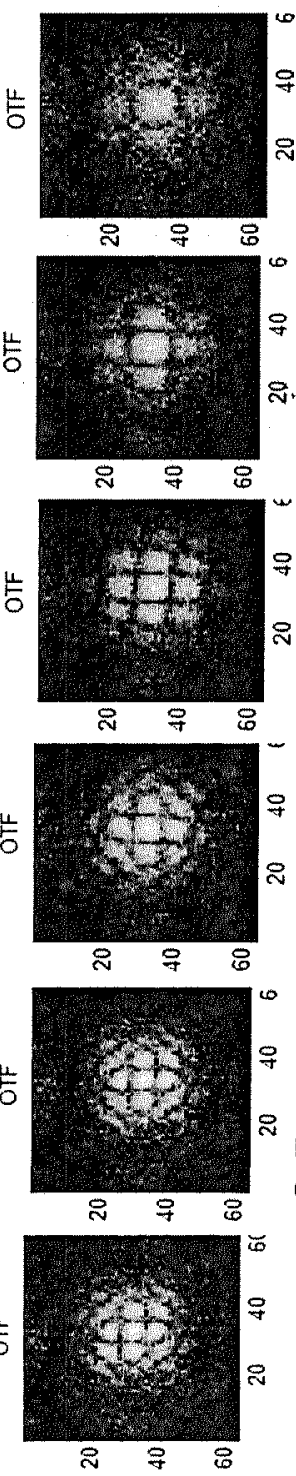
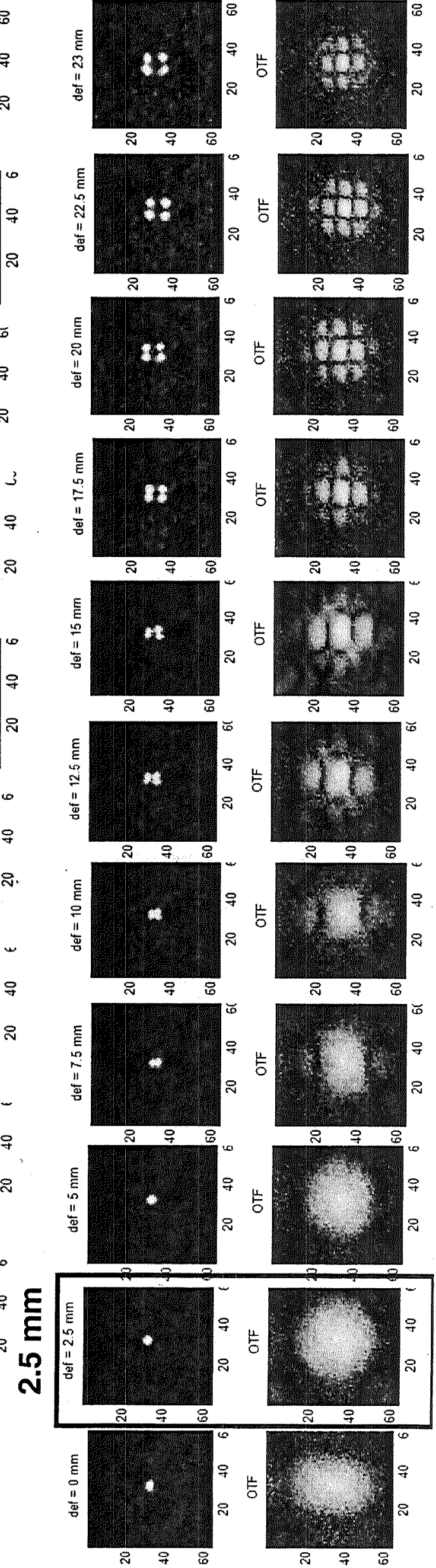


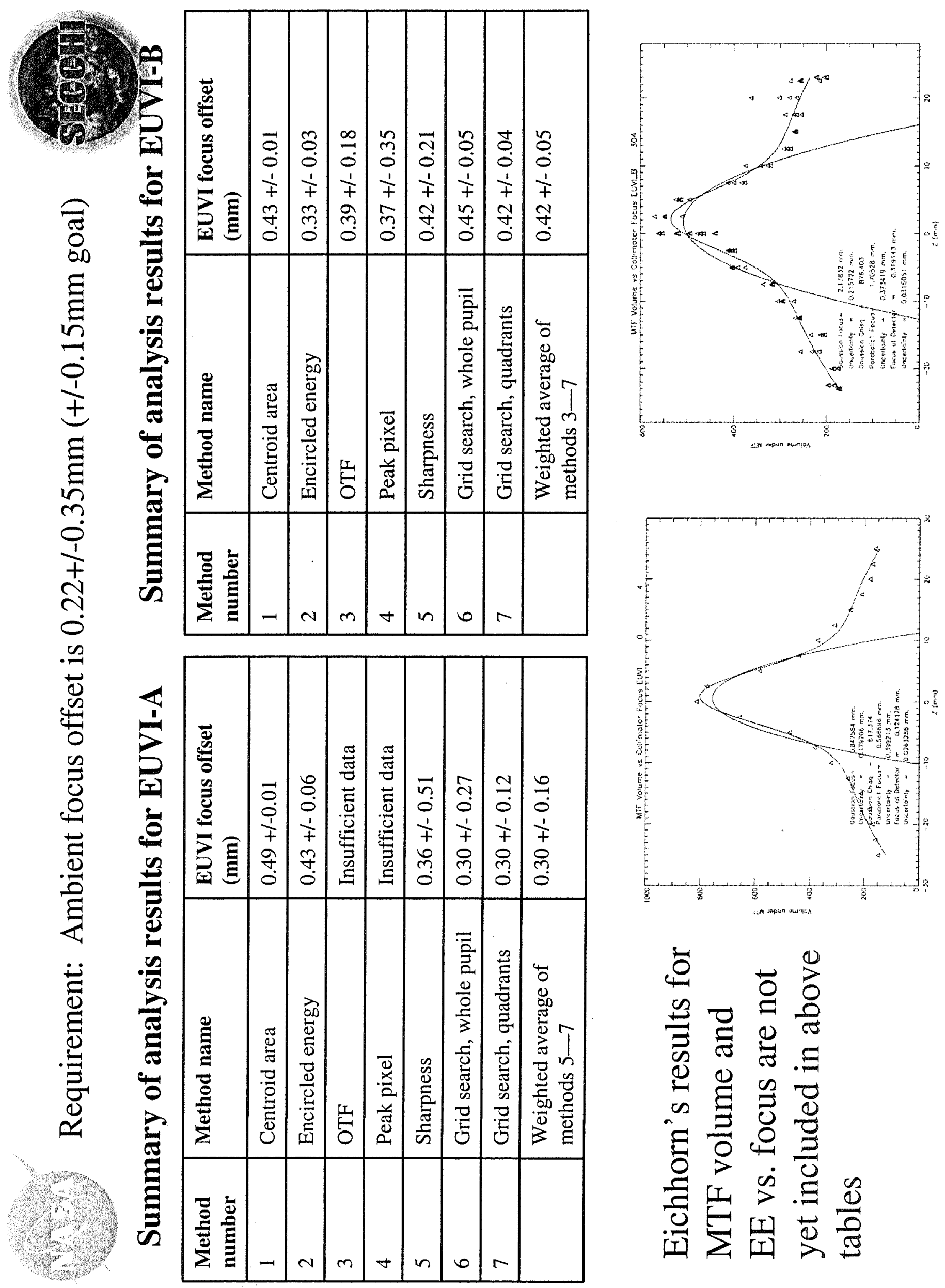



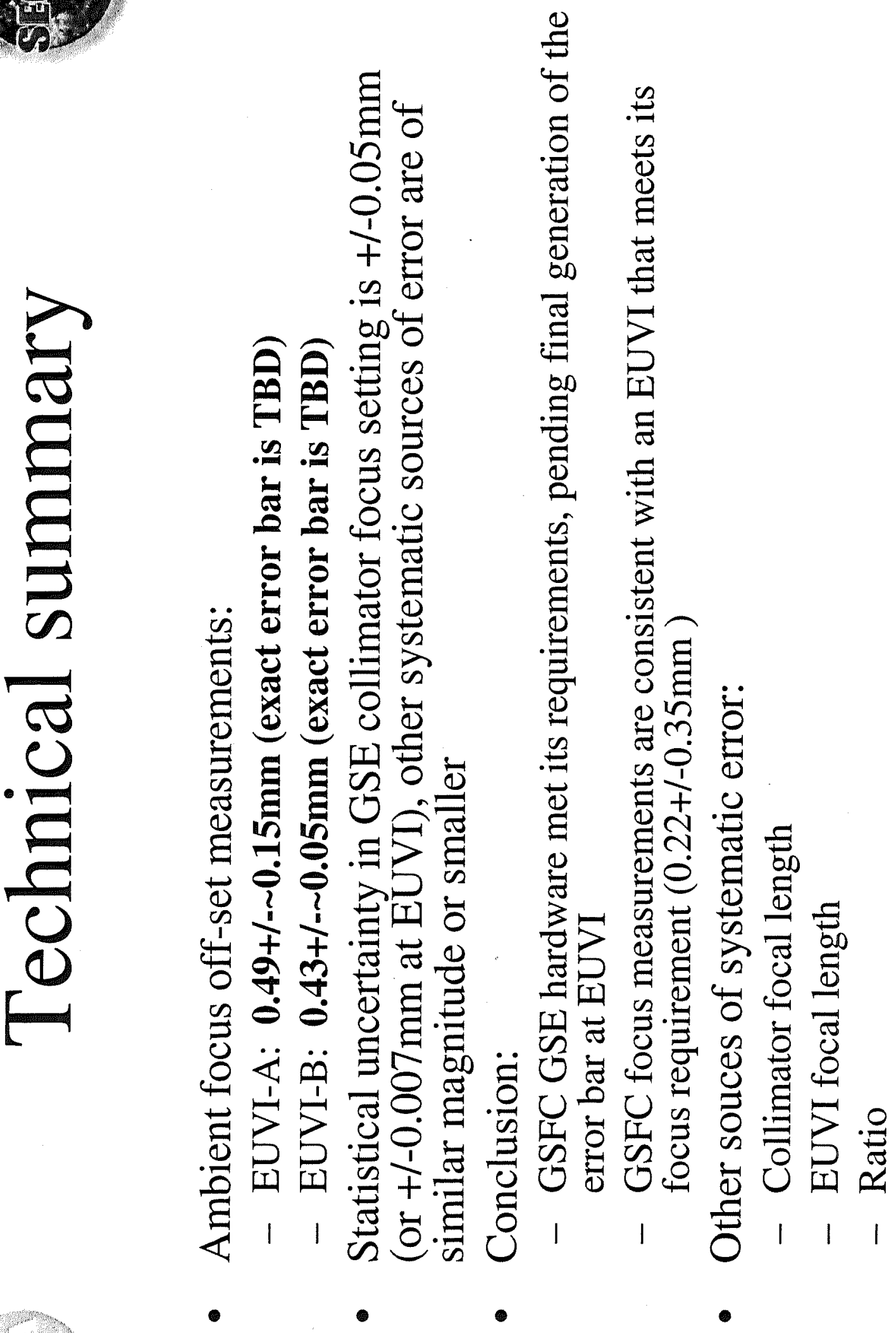

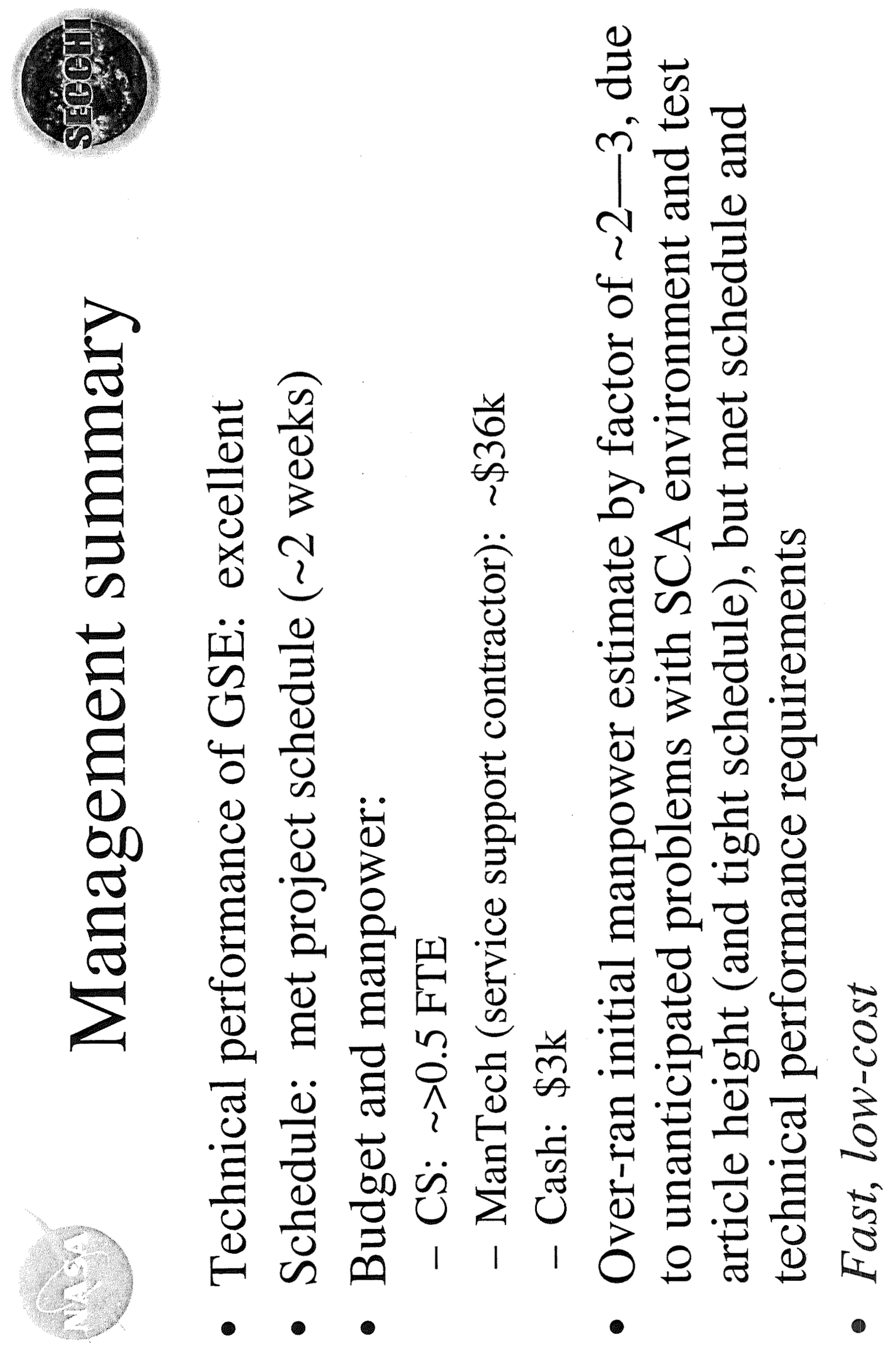

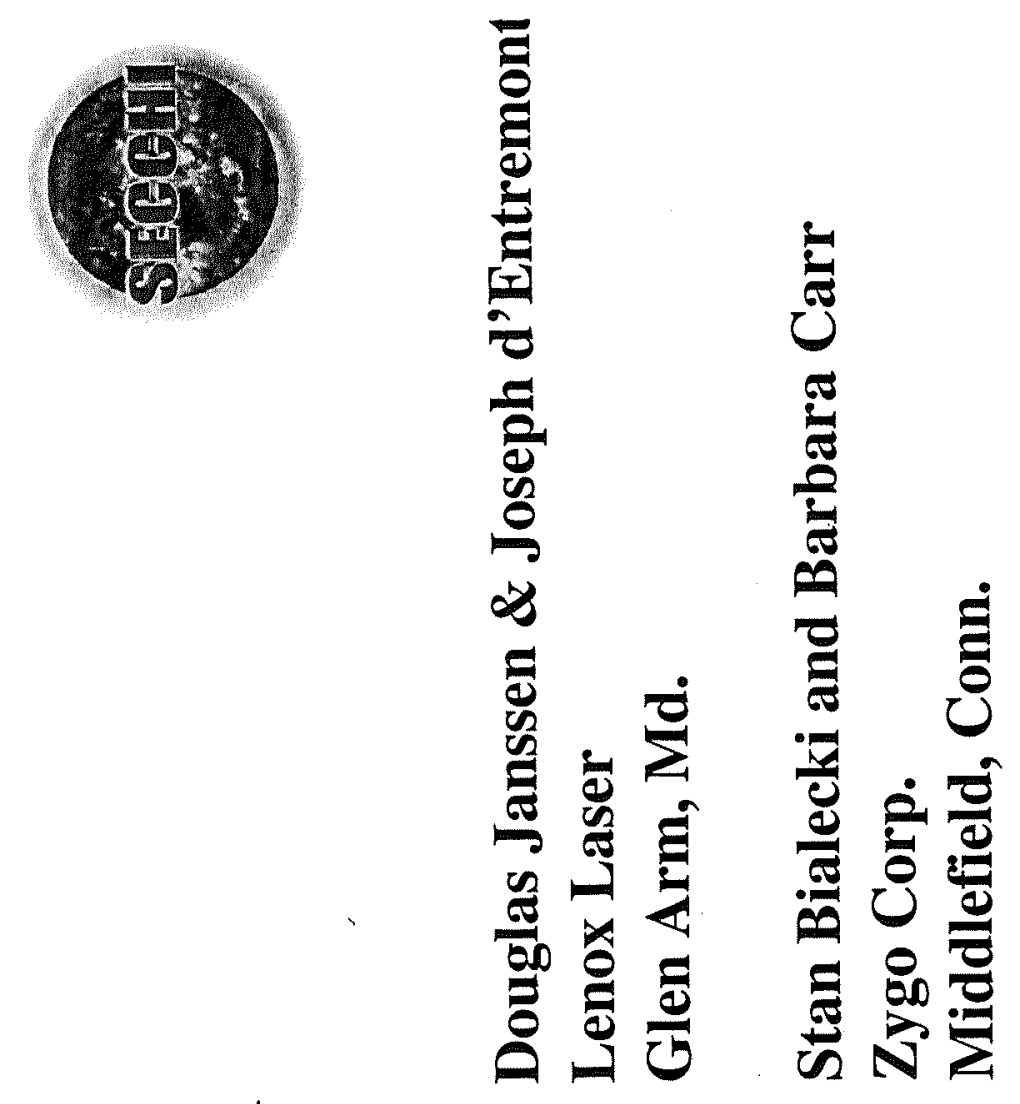

$\frac{8}{0}$
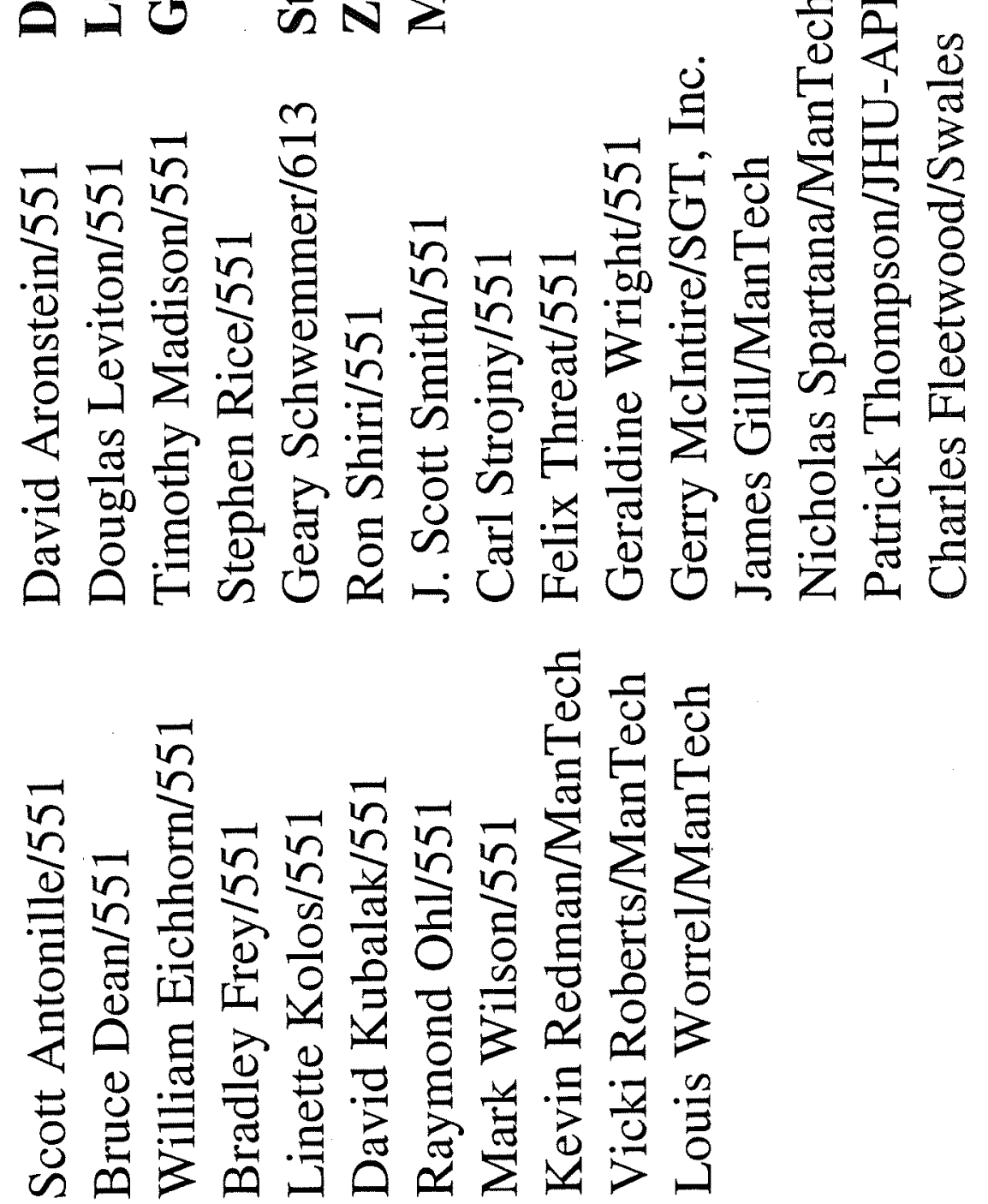


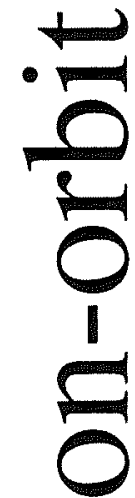

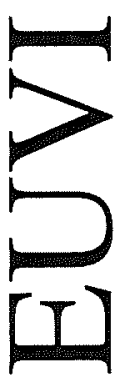

$\square$

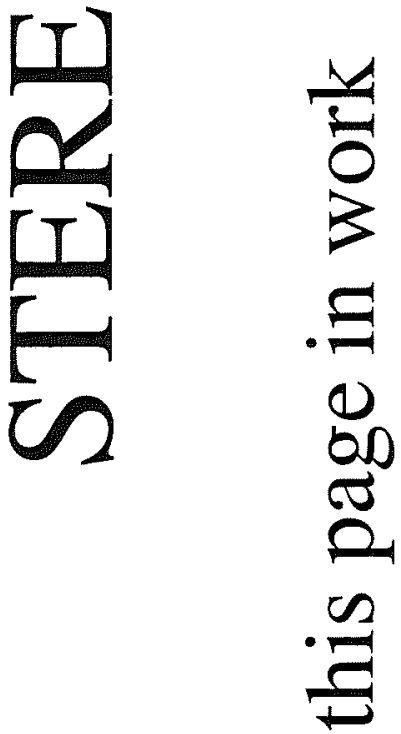

